\title{
Null Controllability and Finite Time Stabilization for the Heat Equations with Variable Coefficients in Space in One Dimension via Backstepping Approach
}

\author{
Jean-Michel Coron and Hoai-Minh Nguyen
}

\author{
Communicated by A. BRESSAN
}

\begin{abstract}
Using the backstepping approach we recover the null controllability for the heat equations with variable coefficients in space in one dimension and prove that these equations can be stabilized in finite time by means of periodic time-varying feedback laws. To this end, on the one hand, we provide a new proof of the wellposedness and the "optimal" bound with respect to damping constants for the solutions of the kernel equations; this allows one to deal with variable coefficients, even with a weak regularity of these coefficients. On the other hand, we establish the well-posedness and estimates for the heat equations with a nonlocal boundary condition at one side.
\end{abstract}

Mathematics Subject Classification 93B05 · 93B17 · 93B52

\section{Introduction}

The null controllability of heat equations has been extensively investigated for several decades. This was pioneered in [13] by the moment method. Since then, there have been a few other methods to prove the null controllability of heat equations. One is based on the construction of the fundamental solution as proposed by $[19,25]$. One is based on Carleman estimates, as initiated in [15,24]; see also [12] and references therein for recent results. One, as proposed in [30], is via the

JMC was supported by ERC advanced Grant 266907 (CPDENL) of the 7th Research Framework Programme (FP7).

HMN was supported by ERC advanced Grant 266907 (CPDENL) of the 7th Research Framework Programme (FP7). 
transmutation method, which relates the null-controllability of the heat equation to the exact controllability of the wave equation. Another, as proposed in [29], is via the flatness approach; in this approach, $x$ is considered as the time-variable, see also [16].

In this paper, we present a new approach to obtain the null controllability for the heat equations with Dirichlet boundary control. This new approach is based on a backstepping design in which the kernel also depends on time. The backstepping method has been used as a standard tool to stabilize finite dimensional control systems, see, e.g., [5,21]. This method was initiated in [6,27] to design feedback laws stabilizing control systems modeled by partial differential equations. Later on, Krstic and his collaborators introduced a key modification of the method: using a Volterra transform of the second kind, coming from the application of the classical backstepping method applied to a spatial discretization of the partial differential equation, they mapped the original equation into an asymptotically stable one. In this context, the first continuous backstepping designs were proposed for the heat equation in $[26,33]$. The applications to wave equations appeared later in $[20,32,35]$. Since then, this has been applied to study the stability of parabolic equations in [33,34], of hyperbolic systems in $[9,10,17,18,22]$, of nonlinear parabolic equations in [36], of Korteweg-de Vries equations in [4] and of Kuramoto-Sivashinsky equations in [28]. A concise introduction to this method applied to numerous partial differential equations can be found in [23]. In this paper, we implement the backstepping idea to obtain the null-controllability of $1-\mathrm{d}$ heat equations. We also provide a new method to prove the existence of and to establish the "optimal" bound for a solution to the kernel equation. Finally, we show how this approach can be used to stabilize in finite time these equations by means of time-varying feedback laws. To this end, we establish the well-posedness and provide estimates for the heat equations with a nonlocal boundary condition at one side.

Remark 1. For some equations, one needs to use a more general transformations than the one given by the backstepping approach (i.e. a Volterra transform of the second kind). See, in particular, [2] for wave equations, [3] for compensating the distributed effect of diffusion and counter-convection in Multi-Input and Multi-Output LTI systems, [7] for KdV equations, [8] for Kuramoto-Sivashinsky equations. In the last two papers, the existence of these more general transformations is shown to be equivalent to the controllability (of the linearized control system).

We consider the control system

$$
\begin{cases}u_{t}(t, x)=\left(a(x) u_{x}(t, x)\right)_{x}+c(x) u(t, x) & \text { in } \quad\left(\tau_{1}, \tau_{2}\right) \times[0,1] \\ u(t, 0)=0, \quad u(t, 1)=U(t) & \text { for } t \in\left(\tau_{1}, \tau_{2}\right)\end{cases}
$$

where, at $t \in\left(\tau_{1}, \tau_{2}\right)$, the state is $u(t, \cdot) \in L^{2}(0,1)$ and the control is $U(t) \in \mathbb{R}$. Throughout this paper, we assume that $a \in H^{2}(0,1), c \in H^{1}(0,1)$, and

$$
a(x)>0 \text { for every } x \in[0,1]
$$


which implies the existence of $\Lambda \geq 1$ such that $0<1 / \Lambda \leq a(x) \leq \Lambda$ in $[0,1]$ since $a$ is continuous on $[0,1]$.

The first goal of this paper is to provide a new way (via backstepping design) to obtain constructive controls $U$ which steer the control system (1.1) from a given $u_{0} \in L^{2}(0,1)$ to 0 during the interval of time $[0, T]$, with $T>0$ given, i.e., $u(t, \cdot) \rightarrow 0$ as $t \rightarrow T_{-}$where $u$ is the solution of

$$
\begin{cases}u_{t}(t, x)=\left(a(x) u_{x}(t, x)\right)_{x}+c(x) u(t, x) & \text { in } \quad(0, T) \times[0,1], \\ u(t, 0)=0, \quad u(t, 1)=U(t) & \text { for } t \in(0, T), \\ u(t=0, \cdot)=u_{0} & \text { for } \quad x \in[0,1] .\end{cases}
$$

Let us denote by $L^{2}(0,1)^{*}$ the set of continuous linear maps from $L^{2}(0,1)$ into $\mathbb{R}$. We have the following theorem:

Theorem 1. Let $T>0$. There exists a piecewise constant functional $\mathcal{K}:[0, T) \rightarrow$ $L^{2}(0,1)^{*}$ such that, for every $u_{0} \in L^{2}(0,1)$, if $u \in C^{0}\left([0, T) ; L^{2}(0,1)\right)$ is the solution of (1.3) with $U(t)$ defined by

$$
U(t):=\mathcal{K}(t) u(t, \cdot),
$$

then

$$
\begin{aligned}
u(t, \cdot) & \rightarrow 0 \text { in } L^{2}(0,1) \text { as } t \rightarrow T_{-}, \\
U(t) & \rightarrow 0 \text { as } t \rightarrow T_{-},
\end{aligned}
$$

where $u$ is the solution of (1.3).

Remark 2. The well-posedness of (1.3) where $U(t)$ is given by (1.4) is established under a more general assumption on $\mathcal{K}$ in Lemma 6 via the maximum principle and the multiplier technique.

Remark 3. Theorem 1 is a consequence of Proposition 1 presented later. More information on $\mathcal{K}$ can be derived from there.

Note that $\mathcal{K}$ must be time dependent. This is the difference between the feedback for the null-controllability in finite time and the feedback for the exponential stability for the heat equation. In fact, assume that $a=1, c=0$, and $\mathcal{K}(t)$ is independent of $t$. There exists $\theta \in L^{2}(0,1)$ such that

$$
\mathcal{K}(t) v=\int_{0}^{1} \theta(x) v(x) \mathrm{d} x \text { for } v \in L^{2}(0,1), t \in(0, T) .
$$

Set, for $\mu \in \mathbb{R}$,

$$
u_{\mu}(t, x)=e^{-\mu^{2} t} \sin (\mu x) .
$$

Note that

$$
\lim _{\mu \rightarrow+\infty} \int_{0}^{1} \theta(x) \sin (\mu x) \mathrm{d} x=0 .
$$


It follows that there exists $\mu \in \mathbb{R} \backslash\{0\}$ such that

$$
\sin \mu=\int_{0}^{1} \theta(x) \sin (\mu x) d x .
$$

One can easily check that $u_{\mu}$ is a solution of the system

$$
\begin{cases}u_{t}-u_{x x}=0 & \text { for } \quad(t, x) \in(0, T) \times(0,1) \\ u(t, 0)=0, & u(t, 1)=\int_{0}^{1} \theta(x) u(t, x) \mathrm{d} x \quad \text { for } \quad 0<t<T .\end{cases}
$$

Hence the null controllability is not achieved with the time invariant feedback $\mathcal{K}$.

Let us briefly describe here the idea of the proof of Theorem 1 . The operator $\mathcal{K}(t)$ in Theorem 1 is of the form

$$
\mathcal{K}(t) v=\int_{0}^{1} k_{n}(1, y) v(y) \mathrm{d} y \quad \text { for every } v \in L^{2}(0,1),
$$

for $t_{n} \leq t<t_{n+1}$ for some sequence $\left(t_{n}\right)_{n \in \mathbb{N}} \rightarrow T_{-}$and for some sequence of functions (called kernels) $\left(k_{n}\right)_{n \in \mathbb{N}}$ defined in $D$ which is given by

$$
D:=\left\{(x, y) \in[0,1]^{2} ; y \leq x\right\}
$$

The choice of $\left(t_{n}\right)_{n \in \mathbb{N}}$ and $\left(k_{n}\right)_{n \in \mathbb{N}}$ are derived from the backstepping approach as follows. Let $\left(t_{n}\right)_{n \in \mathbb{N}}$ be a strictly increasing sequence of real numbers such that $t_{0}=0$ and $t_{n} \rightarrow T$ as $n \rightarrow \infty$. We construct the kernel $k_{n}$ (used for the interval of time $\left.\left[t_{n}, t_{n+1}\right)\right)$ using backstepping design: define, for $t_{n} \leq t<t_{n+1}$,

$$
w(t, x)=u(t, x)-\int_{0}^{x} k_{n}(x, y) u(t, y) \mathrm{d} y,
$$

where $k_{n}$ is chosen such that, for $t_{n} \leq t<t_{n+1}$ and for $u$ solution of (1.1),

$$
w_{t}(t, x)-\left(a(x) w_{x}(t, x)\right)_{x}+\lambda_{n} w(t, x)=0 \quad \text { for } x \in[0,1],
$$

for some $\lambda_{n}>0$, damping coefficients. By requiring that (1.10) holds for any $u$ satisfying $(1.1)$ with $\left(\tau_{1}, \tau_{2}\right)=\left(t_{n}, t_{n+1}\right)$, one obtains the following system for $k_{n}$ :

$$
\begin{cases}2 a(x) \frac{\mathrm{d}}{\mathrm{d} x} k_{n}(x, x)+a_{x}(x) k_{n}(x, x)+\left[\lambda_{n}+c(x)\right]=0 & \text { for } \quad x \in[0,1], \\ k_{n}(x, 0)=0 & \text { for } \quad x \in[0,1], \\ \left(a(x) k_{n, x}(x, y)\right)_{x}-\left(a(y) k_{n, y}(x, y)\right)_{y}-\left[\lambda_{n}+c(y)\right] k_{n}(x, y)=0 & \text { in } \quad D .\end{cases}
$$

Here and in what follows, we use the notation

$$
\frac{\mathrm{d}}{\mathrm{d} x} k(x, x):=k_{x}(x, x)+k_{y}(x, x)
$$

where $k_{x}$ and $k_{y}$ denotes the partial derivative of $k: D \rightarrow \mathbb{R}$ with respect to $x$ and $y$. In fact, we can verify that such a $k_{n}$ exists (Lemma 2) and that, indeed, (1.10) 
holds if $u$ satisfies (1.1) with $\left(\tau_{1}, \tau_{2}\right)=\left(t_{n}, t_{n+1}\right)$ and if $w$ is defined by (1.9) with $k_{n}$ satisfying (1.11) (see Lemma 3). The control $U$ in (1.4) is chosen as usual by requiring (1.8) in order to have

$$
w(t, 1)=0 \text { for } t_{n}<t<t_{n+1} .
$$

Let us point out that, from (1.1) and (1.9), we have

$$
w(t, 0)=0 \text { for } t_{n}<t<t_{n+1} .
$$

We derive from (1.10), (1.12) and (1.13) that

$$
\|w(t, \cdot)\|_{L^{2}} \leq e^{-\lambda_{n}\left(t-t_{n}\right)}\left\|w\left(t_{n+}, \cdot\right)\right\|_{L^{2}} .
$$

From (1.14), we obtain the decay of $w$ as $t \rightarrow T_{-}$. To compute $u$ from $w$, one searches the kernel $l_{n}$ such that

$$
u(t, x)=w(t, x)+\int_{0}^{x} l_{n}(x, y) w(t, y) \mathrm{d} y .
$$

By requiring that, for $t_{n} \leq t<t_{n+1}$,

$$
u_{t}(t, x)=\left(a(x) u_{x}(t, x)\right)_{x}+c(x) u(t, x) \text { for } x \in[0,1],
$$

one gets

$$
\begin{cases}2 a(x) \frac{\mathrm{d}}{\mathrm{d} x} l_{n}(x, x)+a_{x}(x) l_{n}(x, x)+\lambda_{n}+c(x)=0 & \text { for } \quad x \in[0,1], \\ l_{n}(x, 0)=0 & \text { for } \quad x \in[0,1], \\ \left(a(x) l_{n, x}(x, y)\right)_{x}-\left(a(y) l_{n, y}(x, y)\right)_{y}+\left[\lambda_{n}+c(x)\right] l_{n}(x, y)=0 & \text { in } \quad D .\end{cases}
$$

In fact, we can prove that $l_{n}$ exists (Lemma 2) and that, if $l_{n}$ satisfies (1.17) and if $w$ is defined by (1.9) where $k_{n}$ satisfies (1.11), then (1.15) holds (Lemma 4). We establish the following crucial estimates for $k_{n}$ and $l_{n}$ (Lemma 2):

$$
\left\|k_{n}\right\|_{H^{1}(D)} \leq e^{C \lambda_{n}^{1 / 2}} \text { and }\left\|l_{n}\right\|_{H^{1}(D)} \leq C \lambda_{n}^{2},
$$

for some positive constant $C$ which depends only on $a$ and $c$; it does not depend on $n$. Let us point out that related estimates already appear in [24, Proposition 1]. These related estimates are proved thanks to Carleman estimates. Our appoach is completely different. From (1.14) and (1.18), we can derive that $u(t, \cdot) \rightarrow 0$ in $L^{2}(0,1)$ and $U(t) \rightarrow 0$ in $\mathbb{R}$ as $t \rightarrow T_{-}$by appropriate choices of $\left(t_{n}\right)_{n \in \mathbb{N}}$ and of $\left(\lambda_{n}\right)_{n \in \mathbb{N}}$ (see also [24, pp. 343-344]). More precisely, we prove the following proposition:

Proposition 1. Let $T>0,\left(\lambda_{n}\right)_{n \geq 0}$ be an increasing sequence of positive numbers converging to infinity, and let $\left(t_{n}\right)_{n \geq 0}$ be an increasing sequence which converges to $T$ with $t_{0}=0$. Define, for $t_{n} \leq t<t_{n+1}$,

$$
U(t):=\int_{0}^{1} k_{n}(1, y) u(t, y) \mathrm{d} y,
$$


where $k_{n}$ is given in (1.11). Set $s_{0}:=0$ and $s_{n}:=\sum_{k=0}^{n-1} \lambda_{k}\left(t_{k+1}-t_{k}\right)$ for $n \geq 1$. There exists a positive constant $\gamma$, depending only on a and $c$, such that if, for large $n$,

$$
\left(t_{n+1}-t_{n}\right) \lambda_{n} \geq \gamma \sqrt{\lambda_{n+1}},
$$

then, for $t_{n} \leq t \leq t_{n+1}$,

$$
\begin{aligned}
\|u(t, \cdot)\|_{L^{2}} & \leq C e^{-s_{n-1} / 4+C(n-1)}\left\|u_{0}\right\|_{L^{2}}, \\
|U(t)| & \leq C e^{-s_{n-1} / 4+C(n-1)+C \sqrt{\lambda_{n}}}\left\|u_{0}\right\|_{L^{2}},
\end{aligned}
$$

for some positive constant $C$ independent of $n$ and $u_{0}$. In particular, if, in addition we have that

$$
\lim _{n \rightarrow+\infty} \frac{s_{n}}{n+\sqrt{\lambda_{n+1}}}=+\infty
$$

then

$$
\begin{aligned}
\lim _{t \rightarrow T_{-}}\|u(t, \cdot)\|_{L^{2}} & =0, \\
\lim _{t \rightarrow T_{-}} U(t) & =0 .
\end{aligned}
$$

There are sequences $\left(t_{n}\right)_{n \in \mathbb{N}}$ and $\left(\lambda_{n}\right)_{n \in \mathbb{N}}$ which satisfy (1.20) and (1.23), for example, the sequences such that $t_{n}=T-T / n^{2}$ and $\lambda_{n}=n^{8}$ for large $n$. Hence, Theorem 1 is a corollary of Proposition 1.

Remark 4. From the construction of $k_{n}$, one can verify that

$$
\|\mathcal{K}(t)\| \leq C(\tau) \text { for } 0 \leq t \leq \tau<T, \quad \text { and } \quad \lim _{t \rightarrow T_{-}}\|\mathcal{K}(t)\|=+\infty .
$$

It is interesting to know whether or not there exists a bounded feedback $\mathcal{K}$ in $L^{2}(0,1)^{*}$ which yields the null-controllability at time $T$. Note that, in general, one cannot assume too much on the regularity and the boundedness of $\mathcal{K}$. To see this, let us assume that $a=1, c=0$, and $\mathcal{K} \in L^{2}\left((0, T) ; H^{2}(0,1) \cap H_{0}^{1}(0,1)\right)$. For $t \in(0, T)$, let $v(t, \cdot) \in H_{0}^{1}(0,1)$ be the unique solution of $-v_{x x}(t, x)=u(t, x)$ for $x \in(0,1)$. One can check that, for $x \in(0,1)$,

$$
v(t, x)=-\int_{0}^{x} \int_{0}^{y} u(t, s) \mathrm{d} s \mathrm{~d} y+x \int_{0}^{1} \int_{0}^{y} u(t, s) \mathrm{d} s \mathrm{~d} y .
$$

One can then derive that

$$
v_{t}(t, x)-v_{x x}(t, x)=x u(t, 1) .
$$

Since $-v_{x x}(t, x)=u(t, x)$, it follows from integration by parts that

$$
v_{t}(t, x)-v_{x x}(t, x)=-x \int_{0}^{1} \mathcal{K}_{s s}(t, s) v(t, s) \mathrm{d} s .
$$

One now can apply [1, Theorem II.2] to conclude that if $v(T, \cdot)=0$ (this holds if $u(T, \cdot)=0$ ) then $v(0, \cdot)=0$; which in turn implies $u(0, \cdot)=0$. Hence the nullcontrollability is not achieved. More generally, it would be interesting to obtain sharp conditions on $\mathcal{K}$ under which the backward uniqueness holds for (1.3) with $U$ defined in (1.4). 
In this paper, we therefore provide a new constructive control to reach the null controllability for the heat equation with variable coefficients via backstepping approach. Our idea is to use a sequence of kernels $\left(k_{n}\right)_{n \in \mathbb{N}}$ in an appropriate choice of time interval $\left[t_{n}, t_{n+1}\right)$ corresponding for a sequence of positive numbers $\left(\lambda_{n}\right)_{n \in \mathbb{N}}$ used for damping effect converging to infinity to stabilize the equations more and more as $t$ goes to $T$. To implement this idea, we need to prove the existence of $k_{n}$ and $l_{n}$, and establish estimates given in (1.18) (see Corollaries 1 and 2). Our analysis is variational and different from the standard one and hence requires some new ideas. First, to handle the existence of $k_{n}$ and $l_{n}$ in the variable coefficients case, we show, in Lemma 1, a connection of (1.11) and (1.17) with the wave equation defined in $[0,1]^{2}$. To this end, we establish a property of the finite speed of propagation type (see (2.6) in Lemma 1). Known methods to prove the existence of $k_{n}$ and $l_{n}$ (in the case $a$ is constant) are based on special functions or by fixed point arguments (see, in particular, [23, Chap. 4] and [11]). Second, the proof of (1.18) is only known for constants $a$ and $c$ using the information of special functions (see, e.g., [23, Chap. 4]). In the case that $a$ is constant and $c$ is not, it is known that the estimate of $k_{n}$ in (1.18) holds provided that the exponent $\lambda_{n}^{1 / 2}$ is replaced by $\lambda_{n}$ (see, e.g., [23, Chap. 4]). Nevertheless, the exponent $\lambda_{n}$ is not sufficient to get the decay of $u_{n}$ to 0 due to (1.14). Estimates in (1.18) are derived from (2.5) of Lemma 1. Assertion (2.5) is established via an energy type estimate for the wave equation which is somehow nonstandard in the sense that the energy not only contains the gradient of the solutions but also the solutions, see (2.14); the standard energy estimate only gives the exponent $\lambda_{n}$.

The second goal of this article is to show that the control system (1.1) can be semi-globally stabilized in arbitrary time by means of time-varying feedback laws $(t, v) \in \mathbb{R} \times L^{2}(0,1) \mapsto F(t, v) \in \mathbb{R}$. We look for feedback laws $F$ satisfying the following three properties:

$\left(\mathcal{P}_{1}\right)$ The feedback law $F$ is $T$-periodic with respect to time:

$$
F(t, v)=F(t+T, v) \text { for every }(t, v) \in \mathbb{R} \times L^{2}(0,1)
$$

$\left(\mathcal{P}_{2}\right)$ There exists a strictly increasing sequence $\left(t_{n}\right)_{n \in \mathbb{N}}$ of real numbers such that

$$
\begin{aligned}
& t_{0}=0, \\
& \lim _{n \rightarrow+\infty} t_{n}=T, \\
& F \text { is of class } C^{1} \text { in }\left[t_{n}, t_{n+1}\right) \times L^{2}(0,1) \text { for every } n \in \mathbb{N} ;
\end{aligned}
$$

$\left(\mathcal{P}_{3}\right)$ The map $F$ vanishes on $\mathbb{R} \times\{0\}$ and there exists a continuous function $M:[0, T) \rightarrow[0,+\infty)$ such that

$$
\begin{aligned}
& \left|F\left(t, v_{2}\right)-F\left(t, v_{1}\right)\right| \leq M(t)\left\|v_{2}-v_{1}\right\|_{L^{2}} \\
& \quad \forall\left(t, v_{1}, v_{2}\right) \in[0, T) \times L^{2}(0,1) \times L^{2}(0,1) .
\end{aligned}
$$

Before stating our second result, let us make some comments on the Cauchy problem 


$$
\begin{cases}u_{t}(t, x)=\left(a(x) u_{x}(t, x)\right)_{x}+c(x) u(t, x) & \text { for } \quad(t, x) \in(s, \tau) \times[0,1], \\ u(t, 0)=0, \quad u(t, 1)=F(t, u(t, \cdot)) & \text { for } \quad t \in(s, \tau), \\ u(s, \cdot)=u_{0} & \text { for } \quad x \in[0,1],\end{cases}
$$

where $-\infty<s<\tau<+\infty$ and $u_{0} \in L^{2}(0,1)$ are given. We use the following definition: $u:[s, \tau) \times(0,1) \rightarrow \mathbb{R}$ is a solution of $(1.31)$ if $u$ is in $C^{0}\left([s, \tau) ; L^{2}(0,1)\right)$ is such that, for every $\xi \in C^{2}([s, \tau) \times[0,1])$ with compact support in $[s, \tau) \times[0,1]$ and which vanishes on $[s, \tau) \times\{0,1\}$, one has

$$
\begin{aligned}
& -\int_{0}^{1} u_{0}(x) \xi(s, x) \mathrm{d} x-\int_{s}^{\tau} \int_{0}^{1} u(t, x) \xi_{t}(t, x) \mathrm{d} x, \mathrm{~d} t \\
& +\int_{s}^{\tau} a(1) F(t, u(t, \cdot)) \xi_{x}(t, 1) \mathrm{d} t \\
& -\int_{s}^{\tau} \int_{0}^{1} u(t, x)\left(\left(a(x) \xi_{x}(t, x)\right)_{x}+c(x) \xi(t, x)\right) \mathrm{d} x=0 .
\end{aligned}
$$

Using properties $\left(\mathcal{P}_{1}\right)$ and $\left(\mathcal{P}_{3}\right)$, one gets the uniqueness of the solution to the Cauchy problem (1.31): two solutions $u_{1}:\left[s, \tau_{1}\right) \times(0,1) \rightarrow \mathbb{R}$ and $u_{2}:\left[s, \tau_{2}\right) \times(0,1) \rightarrow$ $\mathbb{R}$ to $(1.31)$ are equal on $\left[s, \min \left\{\tau_{1}, \tau_{2}\right\}\right) \times(0, L)$ (see the proof of Lemma 6$)$. A solution $u_{1}:\left[s, \tau_{1}\right) \times(0,1) \rightarrow \mathbb{R}$ to the Cauchy problem (1.31) is said to be maximal if there is no solution $u_{2}:\left[s, \tau_{2}\right) \times(0,1) \rightarrow \mathbb{R}$ to the Cauchy problem (1.31) with $\tau_{2}>\tau_{1}$ and $u_{1}=u_{2}$ on $\left[s, \tau_{1}\right) \times(0,1)$. From now on, all the solutions to the Cauchy problem (1.31) considered are maximal. As was just mentioned, this solution is unique. We denote this solution by $t \in\left[s, \mathfrak{t}\left(s, u_{0}\right)\right) \rightarrow \Phi\left(t, s, u_{0}\right)$. Let us point out that, as proved in Lemma 6, Properties $\left(\mathcal{P}_{1}\right),\left(\mathcal{P}_{2}\right)$ and $\left(\mathcal{P}_{3}\right)$ imply that $\mathfrak{t}\left(s, u_{0}\right)>s$ for every $\left(s, u_{0}\right) \in \mathbb{R} \times L^{2}(0,1)$.

Our second result states that the control system (1.1) can be semi-globally stabilized in arbitrary time by means of time-varying feedback laws $(t, v) \in \mathbb{R} \times$ $L^{2}(0,1) \mapsto F(t, v) \in \mathbb{R}$ satisfying Properties $\left(\mathcal{P}_{1}\right),\left(\mathcal{P}_{2}\right)$ and $\left(\mathcal{P}_{3}\right)$.

Theorem 2. Let $T>0$ and $\Gamma>0$. There exists a time-varying feedback law $(t, v) \in \mathbb{R} \times L^{2}(0,1) \mapsto F(t, v) \in \mathbb{R}$ satisfying Properties $\left(\mathcal{P}_{1}\right),\left(\mathcal{P}_{2}\right)$ and $\left(\mathcal{P}_{3}\right)$ such that

$$
\begin{aligned}
& \exists(C, \bar{T}) \in(0,+\infty) \times(0, T) \text { such that }|F(t, v)| \\
& \quad \leq C\|v\|_{L^{2}}^{1 / 2} \forall(t, v) \in[\bar{T}, T) \times L^{2}(0,1), \\
& \mathfrak{t}\left(s, u_{0}\right)=+\infty \text { for every }\left(s, u_{0}\right) \in \mathbb{R} \times L^{2}(0,1), \\
& \Phi\left(t+2 T, t, u_{0}\right)=0 \text { for every }\left(t, u_{0}\right) \in \mathbb{R} \times L^{2}(0,1) \text { such that }\left\|u_{0}\right\|_{L^{2}} \leq \Gamma,
\end{aligned}
$$

and such that the uniform stability condition

$$
\left\{\begin{array}{l}
\forall \varepsilon>0, \exists \eta>0 \text { such that }, \forall t^{\prime} \in \mathbb{R}, \forall t \in\left[t^{\prime},+\infty\right), \text { and } \forall u_{0} \in L^{2}(0,1), \\
\left(\left\|u_{0}\right\|_{L^{2}} \leq \eta\right) \Rightarrow\left(\left\|\Phi\left(t, t^{\prime}, u_{0}\right)\right\| \leq \varepsilon\right)
\end{array}\right.
$$

holds. 
The paper is organized as follows. In Sect. 2, we establish several lemmas which are used in the proof of Proposition 1 and Theorem 2. In particular, we prove the existence and uniqueness of $k_{n}$ and $l_{n}$, together with estimates for these functions. The proof of Proposition 1 is given in Sect. 3. (Let us recall that, as was observed above, Proposition 1 implies Theorem 1.) In Sect. 4, we establish the well-posedness of (1.31) and various estimates for the flow $\Phi$. Finally, in Sect. 5, we give the proof of Theorem 2.

\section{Preliminaries}

In this section, we establish several lemmas used in the proof of Proposition 1 and Theorem 2. The first one is on the stability and a property of the wave equation, which plays an important role in our analysis and is interesting in itself.

Lemma 1. Let $\lambda \in \mathbb{R}, f \in L^{2}\left((0,1)^{2}\right)$, and let $a_{1}, a_{2}, b_{1}, b_{2}$ and $c$ be bounded measurable functions defined in $[0,1]^{2}$ such that $a_{1}$ and $a_{2}$ are Lipschitz and

$$
\begin{aligned}
& 1 / \Lambda \leq a_{1}(x, y), a_{2}(x, y) \leq \Lambda \text { and } \\
& |b(x, y)|,|c(x, y)| \leq \Lambda \text { for }(x, y) \in[0,1]^{2},
\end{aligned}
$$

for some $\Lambda \geq 1$, where $b=\left(b_{1}, b_{2}\right)$. There exists a unique solution

$$
K \in L^{2}\left((0,1) ; H_{0}^{1}(0,1)\right) \cap H^{1}\left((0,1)^{2}\right)
$$

to the equation

$$
\begin{aligned}
& \left(a_{1}(x, y) K_{x}(x, y)\right)_{x}-\left(a_{2}(x, y) K_{y}(x, y)\right)_{y} \\
& \quad+b(x, y) \cdot \nabla K(x, y)-[\lambda+c(x, y)] K(x, y)=f(x, y) \text { in }[0,1]^{2},
\end{aligned}
$$

such that

$$
\begin{aligned}
& K(x, 0)=K(x, 1)=0 \text { for } x \in[0,1] \\
& \text { and } K(0, y)=K_{x}(0, y)=0 \text { for } y \in[0,1] .
\end{aligned}
$$

Moreover,

$$
\begin{gathered}
\int_{0}^{1}|\nabla K(x, y)|^{2} \mathrm{~d} y \leq C \max \left\{e^{C \operatorname{sign}(\lambda) \sqrt{|\lambda|}}, 1\right\} \int_{0}^{1} \int_{0}^{1}|f(x, y)|^{2} \mathrm{~d} y \mathrm{~d} x \\
\text { for } x \in[0,1] .
\end{gathered}
$$

Assume in addition that $a_{1}(x, x) \geq a_{2}(x, x)$ for $x \in[0,1]$ and supp $f \subset D$. We have

$$
K(x, y)=0 \text { in }[0,1]^{2} \backslash D .
$$

Here $C$ denotes a positive constant depending only on $\Lambda$ and the Lipschitz constant of $\left(a_{1}, a_{2}\right)$. 
We consider (2.3) and (2.4) as a wave system in which $x$ is seen as time variable and $y$ is seen as space variable and use the following definition for solutions: a function $K:(0,1)^{2} \rightarrow \mathbb{R}$ is said to be a solution of (2.3) and (2.4) if (2.2) is satisfied, (2.3) holds in the distributional sense, and

$$
\begin{aligned}
K(0, \cdot) & =0, \\
K_{x}(0, \cdot) & =0 .
\end{aligned}
$$

(Equality (2.7) is an equality in $L^{2}(0,1)$, while $(2.8)$ is an equality in $H^{-1}(0,1)$ : note that, by (2.2) and (2.3), $K \in H^{2}\left((0,1) ; H^{-1}(0,1)\right)$.)

Remark 5. The exponent $\sqrt{\lambda}$ in (2.5) is optimal. This can be seen in the cases $a_{1}=a_{2}=1, b=(0,0)$ and $c=0$ by using the spectral method to solve the wave equation.

Proof. The existence and uniqueness of $K$ are standard and left to the reader. We next prove (2.5) and (2.6). We only give the proof in the case that $a_{1}, a_{2}, b, c$, and $f$ are smooth and satisfy suitable compatibility conditions (which are automatically satisfied if the support of $f$ is included in a compact subset of $(0,1] \times[0,1])$. The proof in the general case follows by a standard regularizing argument. In the case considered, from the standard regularity theory of the wave equation, it follows that $K$ is smooth. We begin with the proof of (2.5). We only consider the case in which $\lambda>0$ and is large enough. The proof in the other case is similar and even simpler. Multiplying the equation of $K$ by $K_{x}(x, y)$, integrating with respect to $y$ from 0 to 1 , and using an integration by parts, we have

$$
\begin{aligned}
& \int_{0}^{1} \frac{1}{2}\left[\frac{\mathrm{d}}{\mathrm{d} x}\left(a_{1}(x, y) K_{x}^{2}(x, y)\right)+a_{1, x}(x, y) K_{x}^{2}(x, y)+\frac{\mathrm{d}}{\mathrm{d} x}\left(a_{2}(x, y) K_{y}^{2}(x, y)\right)\right. \\
& -a_{2, x}(x, y) K_{y}^{2}(x, y)+2 b(x, y) \cdot \nabla K(x, y) K_{x}(x, y) \\
& \left.-[\lambda+c(x, y)] \frac{\mathrm{d}}{\mathrm{d} x} K^{2}(x, y)\right] \mathrm{d} y=\int_{0}^{1} f(x, y) K_{x}(x, y) \mathrm{d} y .
\end{aligned}
$$

This implies

$$
\begin{aligned}
& \frac{\mathrm{d}}{\mathrm{d} x} \int_{0}^{1}\left[a_{1}(x, y) K_{x}^{2}(x, y)+a_{2}(x, y) K_{y}^{2}(x, y)-\lambda K^{2}(x, y)\right] \mathrm{d} y \\
& =2 \int_{0}^{1} f(x, y) K_{x}(x, y) \mathrm{d} y-\int_{0}^{1}\left[a_{1, x}(x) K_{x}^{2}(x, y)-a_{2, x}(x, y) K_{y}^{2}(x, y)\right. \\
& \left.\quad+2 b(x, y) \cdot \nabla K(x, y) K_{x}(x, y)-2 c(x, y) K_{x}(x, y) K(x, y)\right] \mathrm{d} y .
\end{aligned}
$$

Integrating (2.9) from 0 to $x$, using the ellipticity and the Lipschitz property of $a_{1}$ and $a_{2}$ and the boundedness of $b$ and $c$, we obtain, for $x \in[0,1]$,

$$
\begin{aligned}
& \int_{0}^{1}\left[K_{x}^{2}(x, y)+K_{y}^{2}(x, y)\right] \mathrm{d} y \leq C \int_{0}^{1} \lambda K^{2}(x, y) \mathrm{d} y \\
& +C \int_{0}^{x} \int_{0}^{1}\left[K_{x}^{2}(s, y)+K_{y}^{2}(s, y)\right] \mathrm{d} y \mathrm{~d} s+\|f\|_{L^{2}(0,1)^{2}}^{2}
\end{aligned}
$$


Set

$$
\hat{K}(x, y)=K\left(\lambda^{-1 / 2} x, y\right) \text { for }(x, y) \in\left[0, \lambda^{1 / 2}\right] \times[0,1] .
$$

We derive from (2.10) that, for $x \in\left[0, \lambda^{1 / 2}\right]$,

$$
\begin{aligned}
& \int_{0}^{1}\left[\hat{K}_{x}^{2}(x, y)+\lambda^{-1} \hat{K}_{y}^{2}(x, y)\right] \mathrm{d} y \leq C \int_{0}^{1} \hat{K}^{2}(x, y) \mathrm{d} y \\
& +C \int_{0}^{x} \int_{0}^{1}\left[\hat{K}_{x}^{2}(s, y)+\lambda^{-1} \hat{K}_{y}^{2}(s, y)\right] \mathrm{d} y \mathrm{~d} s+\|f\|_{L^{2}}^{2} .
\end{aligned}
$$

Define

$$
V_{1}(x)=\int_{0}^{1}\left[\hat{K}_{x}^{2}(x, y)+\lambda^{-1} \hat{K}_{y}^{2}(x, y)\right] \mathrm{d} y \quad \text { and } \quad V_{2}(x)=\int_{0}^{1} \hat{K}^{2}(x, y) \mathrm{d} y .
$$

We have

$$
V_{2}^{\prime}(x)=2 \int_{0}^{1} \hat{K}_{x}(x, y) \hat{K}(x, y) \mathrm{d} y \leq 2 V_{1}^{1 / 2}(x) V_{2}^{1 / 2}(x),
$$

and from (2.11) we obtain

$$
V_{1}(x) \leq C\left(V_{2}(x)+\int_{0}^{x} V_{1}(s) \mathrm{d} s+\|f\|_{L^{2}}^{2}\right) .
$$

A combination of (2.12) and (2.13) yields

$$
V_{1}(x)+V_{2}^{\prime}(x) \leq C\left(V_{2}(x)+\int_{0}^{x} V_{1}(s) \mathrm{d} s+\|f\|_{L^{2}}^{2}\right) .
$$

We derive from Gronwall's inequality that

$$
\int_{0}^{x} V_{1}(s) \mathrm{d} s+V_{2}(x) \leq C\|f\|_{L^{2}}^{2} e^{C x}
$$

which implies

$$
\int_{0}^{1}\left[\hat{K}_{x}^{2}(x, y)+\lambda^{-1} \hat{K}_{y}^{2}(x, y)\right] \mathrm{d} y \leq C\|f\|_{L^{2}}^{2} e^{C x} .
$$

Estimate (2.5) now follows by a change of variables and the definition of $\hat{K}$.

We next establish that $K(x, y)=0$ in $[0,1]^{2} \backslash D$. Define

$$
E(x)=\frac{1}{2} \int_{x}^{1}\left(a_{1}(x, y) K_{x}^{2}(x, y)+a_{2}(x, y) K_{y}^{2}(x, y)\right) \mathrm{d} y .
$$

We have

$$
\begin{aligned}
E^{\prime}(x)= & \int_{x}^{1}\left(a_{1}(x, y) K_{x x}(x, y) K_{x}(x, y)+\frac{1}{2} a_{1, x}(x, y) K_{x}^{2}(x, y)\right) \mathrm{d} y \\
& +\int_{x}^{1}\left(a_{2}(x, y) K_{y}(x, y) K_{x y}(x, y)+\frac{1}{2} a_{2, x}(x, y) K_{y}^{2}(x, y)\right) \mathrm{d} y \\
& -\frac{1}{2}\left[a_{1}(x, x) K_{x}^{2}(x, x)+a_{2}(x, x) K_{y}^{2}(x, x)\right] .
\end{aligned}
$$


An integration by parts yields

$$
\begin{aligned}
E^{\prime}(x)= & \int_{x}^{1}\left[\left(a_{1}(x, y) K_{x}(x, y)\right)_{x}-\left(a_{2}(x, y) K_{y}(x, y)\right)_{y}\right] K_{x}(x, y) \mathrm{d} y \\
& +\int_{x}^{1}\left(-\frac{1}{2} a_{1, x}(x, y) K_{x}^{2}(x, y)+\frac{1}{2} a_{2, x}(x, y) K_{y}^{2}(x, y)\right) \mathrm{d} y \\
& -\frac{1}{2}\left[a_{1}(x, x) K_{x}^{2}(x, x)+a_{2}(x, x) K_{y}^{2}(x, x)+2 a_{2}(x, x) K_{x}(x, x) K_{y}(x, x)\right] .
\end{aligned}
$$

Since $a_{1}(x, x) \geq a_{2}(x, x)$, we derive that

$$
E^{\prime}(x) \leq C(\lambda) E(x) .
$$

Since $E(0)=0$, it follows that $E=0$. The proof is complete.

Remark 6. The Lipschitz assumption on $a_{1}$ and $a_{2}$ can be weakened, however the uniqueness does not hold if one only assumes (2.1); see [31, Theorem 9].

Using Lemma 1, we can establish the following lemma:

Lemma 2. Let $\lambda \in \mathbb{R}, g \in H^{2}(0,1)$ with $g(0)=0$ and $c \in L^{2}(D)$. There exists a unique solution $k \in H^{1}(D)$ to the system

$$
\left\{\begin{array}{lr}
k(x, x)=g(x) & \text { for } x \in[0,1], \\
k(x, 0)=0 & \text { for } x \in[0,1], \\
\left(a(x) k_{x}(x, y)\right)_{x}-\left(a(y) k_{y}(x, y)\right)_{y}-[\lambda+c(x, y)] k(x, y)=0 & \text { in } D .
\end{array}\right.
$$

Moreover, for every $x \in[0,1]$,

$$
\int_{0}^{x}|\nabla k(x, y)|^{2} \mathrm{~d} y \leq C \max \left\{e^{C \operatorname{sign}(\lambda) \sqrt{|\lambda|}}, 1+|\lambda|^{2}\right\}\|g\|_{H^{2}}^{2},
$$

for some positive constant $C$ independent of $g, x \in[0,1]$, and $\lambda$.

Proof. We first establish the uniqueness of $k$. Assume that $g=0$. We prove that $k=0$. Note that $(-1 / \sqrt{2}, 1 / \sqrt{2})$ is a normal unit vector of $\Gamma:=\{(x, x) ; x \in(0,1)\}$ and, for $x \in[0,1]$,

$$
\left(a(x) k_{x}(x, x),-a(x) k_{y}(x, x)\right) \cdot(-1 / \sqrt{2}, 1 / \sqrt{2})=-\frac{1}{\sqrt{2}} a(x) \frac{\mathrm{d}}{\mathrm{d} x} k(x, x)=0,
$$

since $g=0$. Extend $k=0$ in $[0,1]^{2} \backslash D$ and still denote the extension by $k$. Then $k \in H^{1}\left((0,1)^{2}\right)$ and, by $(2.17)$,

$$
\left(a(x) k_{x}(x, y)\right)_{x}-\left(a(y) k_{y}(x, y)\right)_{y}-[\lambda+c(x, y)] k(x, y)=0 \text { in }[0,1]^{2} .
$$

It is clear that $k(x, 0)=k(x, 1)=0$ for $x \in[0,1]$, and $k(0, y)=k_{x}(0, y)=0$ for $y \in[0,1]$. Hence $k=0$ by Lemma 1 ; the proof of the uniqueness is complete. To prove the existence of $k$, we proceed as follows: define

$$
\varphi(x, y)=g(x) y / x \text { in } D
$$


and set $k_{1}=k-\varphi$ in $D$. Then

$$
\begin{cases}k_{1}(x, x)=0 & \text { for } x \in[0,1], \\ k_{1}(x, 0)=0 & \text { for } x \in[0,1], \\ \left(a(x) k_{1, x}(x, y)\right)_{x}-\left(a(y) k_{1, y}(x, y)\right)_{y}-[\lambda+c(x, y)] k_{1}(x, y)=f_{1}(x, y) & \text { in } D,\end{cases}
$$

where

$$
f_{1}(x, y)=-\left(a(x) \varphi_{x}(x, y)\right)_{x}+\left(a(y) \varphi_{y}(x, y)\right)_{y}+[\lambda+c(x, y)] \varphi(x, y) \text { in } D .
$$

Since

$$
\begin{aligned}
\varphi_{x}(x, y) & =\left[g^{\prime}(x)-g(x) / x\right] y / x, \quad \varphi_{y}(x, y)=g(x) / x, \\
\varphi_{x x}(x, y) & =-2\left[g^{\prime}(x)-g(x) / x\right] y / x^{2}+g^{\prime \prime}(x) y / x, \quad \varphi_{y y}(x, y)=0,
\end{aligned}
$$

and $0 \leq y \leq x \leq 1$ for $(x, y) \in D$, we have, in $D$,

$$
\begin{aligned}
\left|f_{1}(x, y)\right| \leq & C\left(\left|g^{\prime}(x)-g(x) / x\right| / x+\left|g^{\prime \prime}(x)\right|+|g(x) / x|\right. \\
& +(|c(x, y)|+|\lambda|)|g(x)|) .
\end{aligned}
$$

We claim that

$$
\int_{0}^{1} \frac{1}{x}\left|g^{\prime}(x)-g(x) / x\right|^{2} \mathrm{~d} x \leq C\|g\|_{H^{2}}^{2} .
$$

Assuming the claim, we continue the proof. By (2.21) and (2.22), one has

$$
\left\|f_{1}\right\|_{L^{2}(D)} \leq C(1+|\lambda|)\|g\|_{H^{2}} .
$$

By Lemma 1, there exists $k_{2}$ the unique solution to the system

$$
\left(a(x) k_{2, x}(x, y)\right)_{x}-\left(a(y) k_{2, y}(x, y)\right)_{y}-[\lambda+c(x, y)] k_{2}(x, y)=f_{2}(x, y) \text { in }[0,1]^{2},
$$

$k_{2}(x, 0)=k_{2}(x, 1)=0$ for $x \in[0,1]$, and $k_{2}(0, y)=k_{2, x}(0, y)=0$ for $y=$ $[0,1]$. Here $f_{2}=1_{D} f_{1}$ where $1_{D}$ denotes the characteristic function of $D$. Applying Lemma 1 , we also have

$$
k_{2}(x, y)=0 \text { in }[0,1]^{2} \backslash D .
$$

Hence $k_{1}$ can be chosen as the restriction of $k_{2}$ in $D$ and $k=k_{1}+\varphi$ in $D$. Estimate (2.16) follows immediately from the one of $k_{2}$ obtained from Lemma 1 after using (2.19).

It remains to prove $(2.22)$. We have, since $g(0)=0$, that

$$
\begin{aligned}
g^{\prime}(x)-g(x) / x & =g^{\prime}(x)-\int_{0}^{1} g^{\prime}(t x) \mathrm{d} t \\
& =\int_{0}^{1} \int_{0}^{1} x(1-t) g^{\prime \prime}(t x+s(x-t x)) \mathrm{d} s \mathrm{~d} t .
\end{aligned}
$$


It follows that

$\int_{0}^{1} \frac{1}{x}\left|g^{\prime}(x)-g(x) / x\right|^{2} \mathrm{~d} x \leq \int_{0}^{1} \int_{0}^{1} \int_{0}^{1} x(1-t)^{2}\left|g^{\prime \prime}(t x+s(x-t x))\right|^{2} \mathrm{~d} s \mathrm{~d} t \mathrm{~d} x$.

By a change of variables $y=t x+s x-s t x$ and by using Fubini's theorem, we have

$$
\begin{aligned}
& \int_{0}^{1} \int_{0}^{1} \int_{0}^{1}(1-t)^{2}\left|g^{\prime \prime}(t x+s(x-t x))\right|^{2} \mathrm{~d} s \mathrm{~d} t \mathrm{~d} x \\
& \quad \leq \int_{0}^{1} \int_{0}^{1} \int_{0}^{1} \frac{(1-t)^{2}}{t+s-t s}\left|g^{\prime \prime}(y)\right|^{2} \mathrm{~d} y \mathrm{~d} s \mathrm{~d} t .
\end{aligned}
$$

Since

$$
\int_{0}^{1} \int_{0}^{1} \frac{(1-t)^{2}}{t+s-t s} \mathrm{~d} s \mathrm{~d} t=-\int_{0}^{1}(1-t) \ln t \mathrm{~d} t<+\infty,
$$

the claim (2.22) now follows from (2.24) and (2.25).

From Lemma 2, we get the following corollary, which gives the existence and uniqueness of $k_{n}$ together with the first inequality of (1.18):

Corollary 1. Let $\lambda_{0}>0$. For every $\lambda \geq \lambda_{0}$, there exists a unique solution $k \in$ $H^{1}(D)$ of the system

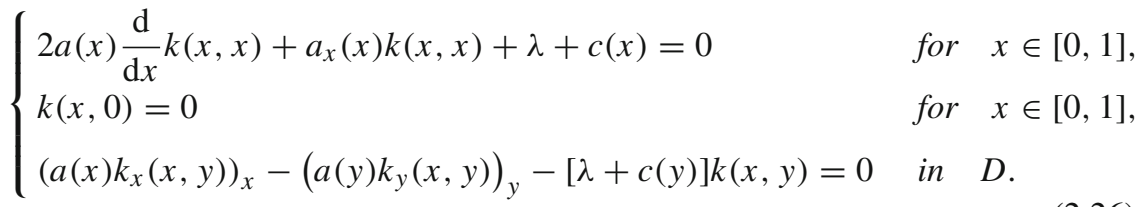

Moreover,

$$
\|k\|_{H^{1}(D)} \leq e^{C \lambda^{1 / 2}}
$$

for some positive constant $C$ independent of $\lambda \in\left[\lambda_{0},+\infty\right)$.

Proof. Since $a \in H^{2}(0,1)$ and $c \in H^{1}(0,1)$, there exists (a unique) $g \in H^{2}(0,1)$ such that

$$
2 a(x) g^{\prime}(x)+a_{x}(x) g(x)+\lambda+c(x)=0 \text { in }[0,1] \quad \text { and } \quad g(0)=0 ;
$$

moreover,

$$
\|g\|_{H^{2}} \leq C \lambda,
$$

for some positive constant $C$ independent of $\lambda$. Then (2.26) is equivalent to

$$
\begin{cases}k(x, x)=g(x) & \text { for } x \in[0,1], \\ k(x, 0)=0 & \text { for } x \in[0,1], \\ \left(a(x) k_{x}(x, y)\right)_{x}-\left(a(y) k_{y}(x, y)\right)_{y}-[\lambda+c(y)] k(x, y)=0 & \text { in } D .\end{cases}
$$

The existence and uniqueness of $k$ now follow from Lemma 2 and estimate (2.27) is a consequence of (2.16) and (2.28). 
Similar to Corollary 1, we obtain the following consequence of Lemma 2 whose proof is left to the reader:

Corollary 2. Let $\lambda_{0}>0$. For every $\lambda \geq \lambda_{0}$, there exists a unique solution $l \in$ $H^{1}(D)$ of the system

$$
\begin{cases}2 a(x) \frac{\mathrm{d}}{\mathrm{d} x} l(x, x)+a_{x}(x) l(x, x)+\lambda+c(x)=0 & \text { for } x \in[0,1], \\ l(x, 0)=0 & \text { for } x \in[0,1], \\ \left(a(x) l_{x}(x, y)\right)_{x}-\left(a(y) l_{y}(x, y)\right)_{y}+[\lambda+c(x)] l(x, y)=0 & \text { in } D .\end{cases}
$$

Moreover,

$$
\|l\|_{H^{1}(D)} \leq C \lambda^{2}
$$

for some positive constant $C$ independent of $\lambda \in\left[\lambda_{0},+\infty\right)$.

Corollary 2 gives the existence and uniqueness of $l_{n}$ together with the second inequality of (1.18).

The third lemma, whose proof is quite standard, is on the link between (1.1) and (1.10).

Lemma 3. Let $\lambda>0$ and $\tau_{2}>\tau_{1} \geq 0$. Assume that $u \in L^{2}\left(\left(\tau_{1}, \tau_{2}\right) ; H^{1}(0,1)\right)$ is a solution to the system

$$
\begin{aligned}
u_{t}(t, x)-\left(a(x) u_{x}(t, x)\right)_{x}-c(x) u(t, x) & =0 \text { in }\left(\tau_{1}, \tau_{2}\right) \times[0,1], \\
u(t, 0) & =0 \text { for } t \in\left(\tau_{1}, \tau_{2}\right) .
\end{aligned}
$$

Define, for $\tau_{1}<t<\tau_{2}$,

$$
w(t, x)=u(t, x)-\int_{0}^{x} k(x, y) u(t, y) \mathrm{d} y,
$$

where $k \in H^{1}(D)$ is the unique solution of the system (2.26). Then $w \in L^{2}\left(\left(\tau_{1}, \tau_{2}\right) ; H^{1}(0,1)\right)$ and satisfies the equation

$$
w_{t}(t, x)-\left(a(x) w_{x}(t, x)\right)_{x}+\lambda w(t, x)=0 \text { in }\left(\tau_{1}, \tau_{2}\right) \times[0,1] .
$$

Proof. We assume that $a$ and $c$ are smooth and establish (2.34); the general case follows by a regularizing argument. The smoothness of $a$ and $c$ imply that $k$ and $u$ and hence $w$ are smooth in $\left(\tau_{1}, \tau_{2}\right) \times[0,1]$. We have, from $(2.33)$,

$$
w_{t}(t, x)=u_{t}(t, x)-\int_{0}^{x} k(x, y) u_{t}(t, y) \mathrm{d} y .
$$

Using the fact that $u_{t}(t, x)=\left(a(x) u_{x}(t, x)\right)_{x}+c(x) u(t, x)$ in $\left(\tau_{1}, \tau_{2}\right) \times[0,1]$ and integrating by parts, we derive from (2.35) that

$$
\begin{aligned}
w_{t}(t, x)= & u_{t}(t, x)-\int_{0}^{x}\left[\left(a(y) k_{y}(x, y)\right)_{y}+c(y) k(x, y)\right] u(t, y) \mathrm{d} y \\
& -\left.k(x, y) a(y) u_{y}(t, y)\right|_{0} ^{x}+\left.a(y) k_{y}(x, y) u(t, y)\right|_{0} ^{x}
\end{aligned}
$$


This implies, by (2.32), that

$$
\begin{aligned}
w_{t}(t, x)= & u_{t}(t, x)-\int_{0}^{x}\left[\left(a(y) k_{y}(x, y)\right)_{y}+c(y) k(x, y)\right] u(t, y) \mathrm{d} y \\
& -k(x, x) a(x) u_{x}(t, x)+a(x) k_{y}(x, x) u(t, x) \\
& +k(x, 0) a(0) u_{x}(t, 0) .
\end{aligned}
$$

From (2.33), we also have

$$
\begin{aligned}
\left(a(x) w_{x}(t, x)\right)_{x}= & \left(a(x) u_{x}(t, x)\right)_{x}-\int_{0}^{x}\left(a(x) k_{x}(x, y)\right)_{x} u(t, y) \mathrm{d} y \\
& -\frac{\mathrm{d}}{\mathrm{d} x}(a(x) k(x, x)) u(t, x)-a(x) k(x, x) u_{x}(t, x) \\
& -a(x) k_{x}(x, x) u(t, x) .
\end{aligned}
$$

Using the fact that $u_{t}(t, x)-\left(a(x) u_{x}(t, x)\right)_{x}-c(x) u(t, x)=0$ in $\left(\tau_{1}, \tau_{2}\right) \times$ $[0,1]$, we derive from $(2.33),(2.36)$ and $(2.37)$ that

$$
\begin{aligned}
& w_{t}(t, x)-\left(a(x) w_{x}(t, x)\right)_{x}+\lambda w(t, x) \\
& =\left[2 a(x) \frac{\mathrm{d}}{\mathrm{d} x} k(x, x)+a_{x}(x) k(x, x)+\lambda+c(x)\right] u(t, x)+k(x, 0) a(0) u_{x}(t, 0) \\
& \quad+\int_{0}^{x}\left[\left(a(x) k_{x}(x, y)\right)_{x}-\left(a(y) k_{y}(x, y)\right)_{y}-[\lambda+c(y)] k(x, y)\right] u(t, y) .
\end{aligned}
$$

With the choice $k$ in $(2.26)$, we have

$$
w_{t}(t, x)-\left(a(x) w_{x}(t, x)\right)_{x}+\lambda w(t, x)=0 \text { in }\left(\tau_{1}, \tau_{2}\right) \times[0,1] .
$$

The proof is complete.

The fourth lemma deals with the inverse transform of the map $u \mapsto w$, where $w$ is defined by (1.9).

Lemma 4. Let $\lambda>0$, let $k \in H^{1}(D)$ be the unique solution of (2.26), and let $l \in H^{1}(D)$ be the unique solution of (2.29). Let $u \in L^{2}(0,1)$ and define

$$
w(x)=u(x)-\int_{0}^{x} k(x, y) u(y) \mathrm{d} y \text { for } x \in[0,1] .
$$

We have

$$
u(x)=w(x)+\int_{0}^{x} l(x, y) w(y) \mathrm{d} y \text { in }[0,1] .
$$

Proof. In what follows, we assume that $a$ and $c$ are smooth, the general case follows by a regularizing argument. Since $a$ and $c$ are smooth, it follows that $k$ and $l$ are smooth. We claim that

$$
l(x, y)=k(x, y)+\int_{y}^{x} l(x, \xi) k(\xi, y) \mathrm{d} \xi .^{1}
$$

\footnotetext{
1 This identity is different from [23, (4.35) on page 38] and is new for us.
} 
Admitting this claim, we prove (2.38). We have, by Fubini's theorem, that

$$
\begin{aligned}
& w(x)+\int_{0}^{x} l(x, y) w(y) \mathrm{d} y \\
& =u(x)-\int_{0}^{x} k(x, y) u(y)+\int_{0}^{x} l(x, y)\left[u(y)-\int_{0}^{y} k(y, \xi) u(\xi) d \xi\right] \mathrm{d} y \\
& =u(x)+\int_{0}^{x}\left[l(x, y)-k(x, y)-\int_{y}^{x} l(x, \xi) k(\xi, y) d \xi\right] u(y) \mathrm{d} y=u(x)
\end{aligned}
$$

(2.38) is proved.

It remains to establish (2.39). Define

$$
\hat{l}(x, y)=k(x, y)+\int_{y}^{x} l(x, \xi) k(\xi, y) \mathrm{d} \xi .
$$

We will prove that $\hat{l}=l$. It is clear that

$\hat{l}(x, x)=k(x, x)=l(x, x)$ and $\hat{l}(x, 0)=k(x, 0)=l(x, 0)=0$ for $x \in[0,1]$.

We have, from (2.40),

$$
\hat{l}_{x}(x, y)=k_{x}(x, y)+\int_{y}^{x} l_{x}(x, \xi) k(\xi, y) d \xi+l(x, x) k(x, y) .
$$

It follows that

$$
\begin{aligned}
\left(a(x) \hat{l}_{x}(x, y)\right)_{x}= & \left(a(x) k_{x}(x, y)\right)_{x}+\int_{y}^{x}\left(a(x) l_{x}(x, \xi)\right)_{x} k(\xi, y) d \xi \\
& +a(x) l_{x}(x, x) k(x, y)+\frac{\mathrm{d}}{\mathrm{d} x}[a(x) l(x, x)] k(x, y) \\
& +a(x) l(x, x) k_{x}(x, y) .
\end{aligned}
$$

Using the fact that $\left(a(x) l_{x}(x, y)\right)_{x}=\left(a(y) l_{y}(x, y)\right)_{y}-[\lambda+c(x)] l(x, y)$ in $D$, we have

$$
\begin{aligned}
\left(a(x) \hat{l}_{x}(x, y)\right)_{x}= & \left(a(x) k_{x}(x, y)\right)_{x}+\int_{y}^{x}\left[\left(a(\xi) l_{\xi}(x, \xi)\right)_{\xi} k(\xi, y)\right. \\
& -[\lambda+c(x)] l(x, \xi) k(\xi, y)] \mathrm{d} \xi+a(x) l_{x}(x, x) k(x, y) \\
& +\frac{\mathrm{d}}{\mathrm{d} x}[a(x) l(x, x)] k(x, y)+a(x) l(x, x) k_{x}(x, y) \\
= & \left(a(x) k_{x}(x, y)\right)_{x}+\int_{y}^{x}\left[l(x, \xi)\left(a(\xi) k_{\xi}(\xi, y)\right)_{\xi}\right. \\
& -[\lambda+c(x)] l(x, \xi) k(\xi, y)] \mathrm{d} \xi+\left.a(\xi) l_{\xi}(x, \xi) k(\xi, y)\right|_{\xi=y} ^{\xi=x} \\
& -\left.l(x, \xi) a(\xi) k_{\xi}(\xi, y)\right|_{\xi=y} ^{\xi=x}+a(x) l_{x}(x, x) k(x, y) \\
& +\frac{\mathrm{d}}{\mathrm{d} x}[a(x) l(x, x)] k(x, y)+a(x) l(x, x) k_{x}(x, y) .
\end{aligned}
$$


This implies

$$
\begin{aligned}
\left(a(x) \hat{l}_{x}(x, y)\right)_{x}= & \left(a(x) k_{x}(x, y)\right)_{x}+\int_{y}^{x}\left[\left(a(\xi) k_{\xi}(\xi, y)\right)_{\xi}\right. \\
& -[\lambda+c(x)] k(\xi, y)] l(x, \xi) \mathrm{d} \xi+a(x) l_{y}(x, x) k(x, y) \\
& -a(y) l_{y}(x, y) k(y, y)+l(x, y) a(y) k_{x}(y, y) \\
& +a(x) l_{x}(x, x) k(x, y)+\frac{\mathrm{d}}{\mathrm{d} x}[a(x) l(x, x)] k(x, y) .
\end{aligned}
$$

Then, using the first equality of (2.29), it follows that

$$
\begin{aligned}
\left(a(x) \hat{l}_{x}(x, y)\right)_{x}= & \left(a(x) k_{x}(x, y)\right)_{x}+\int_{y}^{x}\left[\left(a(\xi) k_{\xi}(\xi, y)\right)_{\xi}\right. \\
& -[\lambda+c(x)] k(\xi, y)] l(x, \xi) \mathrm{d} \xi-[\lambda+c(x)] k(x, y) \\
& -a(y) l_{y}(x, y) k(y, y)+l(x, y) a(y) k_{x}(y, y) .
\end{aligned}
$$

Similarly, from (2.40), we obtain

$$
\hat{l}_{y}(x, y)=k_{y}(x, y)+\int_{y}^{x} l(x, \xi) k_{y}(\xi, y) \mathrm{d} \xi-l(x, y) k(y, y) .
$$

It follows that

$$
\begin{aligned}
\left(a(y) \hat{l}_{y}(x, y)\right)_{y}= & \left(a(y) k_{y}(x, y)\right)_{y}+\int_{y}^{x} l(x, \xi)\left(a(y) k_{y}(\xi, y)\right)_{y} \mathrm{~d} \xi \\
& -a(y) l(x, y) k_{y}(y, y)-l(x, y)(a(y) k(y, y))_{y} \\
& -l_{y}(x, y) a(y) k(y, y) .
\end{aligned}
$$

Using the first and the last equation of (2.26), we derive from (2.42) and (2.43) that

$$
\begin{aligned}
& \left(a(x) \hat{l}_{x}(x, y)\right)_{x}-\left(a(y) \hat{l}_{y}(x, y)\right)_{y}+[c(x)-c(y)](k(x, y) \\
& \left.+\int_{y}^{x} l(x, \xi) k(\xi, y) \mathrm{d} \xi\right)=-[\lambda+c(y)] l(x, y),
\end{aligned}
$$

which yields, by the definition of $\hat{l}$,

$$
\left(a(x) \hat{l}_{x}(x, y)\right)_{x}-\left(a(y) \hat{l}_{y}(x, y)\right)_{y}+[c(x)-c(y)] \hat{l}(x, y)=-[\lambda+c(y)] l(x, y) .
$$

On the other hand, from the last equation of (2.29), we have

$$
\left(a(x) l_{x}(x, y)\right)_{x}-\left(a(y) l_{y}(x, y)\right)_{y}+[c(x)-c(y)] l(x, y)=-[\lambda+c(y)] l(x, y) .
$$

Combing (2.41), (2.44) and (2.45), and using Lemma 2, yields that $\hat{l}=l$. The proof is complete. 


\section{Proof of Proposition 1}

This section is devoted to the proof of Proposition 1. We implement the ideas presented in the introduction. For $t_{n} \leq t<t_{n+1}$, Define $w$ by (1.9) where $k_{n}$ is given in (1.11). Applying Corollary 1, we have

$$
\|w(t, \cdot)\|_{L^{2}}^{2} \leq C e^{C \sqrt{\lambda_{n}}}\|u(t, \cdot)\|_{L^{2}}^{2} \quad \text { for } \quad t_{n}<t<t_{n+1} .
$$

By Lemma 4, (1.15) holds where $l_{n}$ is given by (1.17). We have, by applying Corollary 2,

$$
\|u(t, \cdot)\|_{L^{2}}^{2} \leq C \lambda_{n}^{4}\|w(t, \cdot)\|_{L^{2}}^{2} \quad \text { for } \quad t_{n} \leq t<t_{n+1},
$$

and, by using Lemma 3,

$$
w_{t}(t, x)-\left(a(x) w_{x}(t, x)\right)_{x}+\lambda_{n} w(t, x)=0 \text { for } x \in[0,1] .
$$

From the choice of the control (1.19), we obtain

$$
w(t, 0)=w(t, 1)=0 \text { for } t_{n}<t<t_{n+1} .
$$

It follows that

$$
\left\|w\left(\xi_{2}, \cdot\right)\right\|_{L^{2}}^{2} \leq\left\|w\left(\xi_{1}, \cdot\right)\right\|_{L^{2}}^{2} e^{-2 \lambda_{n}\left(\xi_{2}-\xi_{1}\right)} \quad \text { for } \quad t_{n} \leq \xi_{1}<\xi_{2}<t_{n+1} .
$$

A combination of (3.1), (3.2), and (3.3) yields

$$
\left\|u\left(t_{n+1}, \cdot\right)\right\|_{L^{2}}^{2} \leq C \lambda_{n}^{4} e^{-2 \lambda_{n}\left(t_{n+1}-t_{n}\right)+C \sqrt{\lambda_{n}}}\left\|u\left(t_{n}, \cdot\right)\right\|_{L^{2}}^{2} .
$$

We derive from (1.20) and (3.4) that, if $\gamma$ is large enough, which will be always assumed,

$$
\left\|u\left(t_{n+1}, \cdot\right)\right\|_{L^{2}}^{2} \leq C e^{-\lambda_{n}\left(t_{n+1}-t_{n}\right)}\left\|u\left(t_{n}, \cdot\right)\right\|_{L^{2}}^{2} .
$$

This, together with the definition of $s_{n}$, implies

$$
\left\|u\left(t_{n+1}, \cdot\right)\right\|_{L^{2}}^{2} \leq e^{-s_{n+1}+C n}\|u(0, \cdot)\|_{L^{2}}^{2} .
$$

We have, for $t_{n} \leq t<t_{n+1}$,

$$
\begin{aligned}
\|u(t, \cdot)\|_{L^{2}}^{2} & \leq C \lambda_{n}^{4}\|w(t, \cdot)\|_{L^{2}}^{2} \leq C \lambda_{n}^{4} e^{-2 \lambda_{n}\left(t-t_{n}\right)}\left\|w\left(t_{n}+, \cdot\right)\right\|_{L^{2}}^{2} \\
& \leq C \lambda_{n}^{4} e^{-2 \lambda_{n}\left(t-t_{n}\right)+C \sqrt{\lambda_{n}}}\left\|u\left(t_{n}, \cdot\right)\right\|_{L^{2}}^{2} \\
& \leq C e^{-\lambda_{n-1}\left(t_{n}-t_{n-1}\right) / 2}\left\|u\left(t_{n-1}, \cdot\right)\right\|_{L^{2}}^{2} \\
& \leq e^{-s_{n-1} / 2+C(n-2)}\|u(0, \cdot)\|_{L^{2}}^{2},
\end{aligned}
$$

which gives (1.21). Here we use (3.2) in the first inequality of (3.7), (3.3) in the second inequality, (3.1) in the third inequality, (1.20) and (3.5) in the fourth inequality, and (3.6) (for $t_{n-1}$ instead of $t_{n+1}$ ) in the last inequality. Finally (1.22) follows from (1.18), (1.19) and (1.21). 


\section{Some Properties of the Flow $\Phi$}

In this section, we are interested in the flow $\Phi$ introduced in the Introduction (Sect. 1). We start by mentioning a maximum principle for the Cauchy problem

$$
\begin{cases}u_{t}(t, x)=\left(a(x) u_{x}(t, x)\right)_{x}+c(x) u(t, x)+f(t, x) & \text { in }\left(\tau_{1}, \tau_{2}\right) \times(0,1), \\ u(t, 0)=\alpha(t), \quad u(t, 1)=\beta(t) & \text { for } t \in\left(\tau_{1}, \tau_{2}\right), \\ u(0, x)=u_{0}(x), & \text { for } x \in(0,1),\end{cases}
$$

which we will use many times in this section. Let $\tau_{1}$ and $\tau_{2}$ be two real numbers such that $\tau_{1}<\tau_{2}$, let $\alpha, \beta \in L^{2}\left(\tau_{1}, \tau_{2}\right)$, let $f \in L^{2}\left(\left(\tau_{1}, \tau_{2}\right) \times(0,1)\right)$, and let $u_{0} \in H^{-1}(0,1)$. Let us recall that, with this regularity on $\alpha, \beta$ and $f$, the Cauchy problem (4.1) is well-posed in $C^{0}\left(\left[\tau_{1}, \tau_{2}\right] ; H^{-1}(0,1)\right)$ : it has one and only one solution in this set and there exists a constant $C>0$ independent of $\alpha, \beta \in L^{2}\left(\tau_{1}, \tau_{2}\right), f \in L^{2}\left(\left(\tau_{1}, \tau_{2}\right) \times(0,1)\right)$, and $u_{0} \in H^{-1}(0,1)$ such that

$$
\begin{gathered}
\|u\|_{C^{0}\left(\left[\tau_{1}, \tau_{2}\right], H^{-1}(0,1)\right) \leq} \leq\left(\|\alpha\|_{L^{2}\left(\tau_{1}, \tau_{2}\right)}+\|\beta\|_{L^{2}\left(\tau_{1}, \tau_{2}\right)}+\|f\|_{L^{2}\left(\left(\tau_{1}, \tau_{2}\right) \times(0,1)\right)}\right. \\
\left.+\left\|u_{0}\right\|_{H^{-1}(0,1)}\right) .
\end{gathered}
$$

If $u_{0} \in L^{2}(0,1)$, then the solution is also in $C^{0}\left(\left[\tau_{1}, \tau_{2}\right] ; L^{2}(0,1)\right)$ and the variant of (4.2) with $H^{-1}(0,1)$ replaced by $L^{2}(0,1)$ holds. The notion of a solution to the Cauchy problem (4.1) has to be understood in the transposition sense (compare with (1.32)): a solution $u$ of (4.1) is a function $u$ in $C^{0}\left(\left[\tau_{1}, \tau_{2}\right] ; H^{-1}(0,1)\right)$ such that, for every $s \in\left[\tau_{1}, \tau_{2}\right]$ and for every $\xi \in L^{2}\left(\left(\tau_{1}, s\right) ; H^{2}(0,1)\right) \cap H^{1}\left(\left(\tau_{1}, s\right) ; H_{0}^{1}(0,1)\right)$ such that

$$
-\xi_{t}=\left(a(x) \xi_{x}\right)_{x}+c(x) \xi \text { in } L^{2}\left(\left(\tau_{1}, s\right) \times(0,1)\right)
$$

one has

$$
\begin{gathered}
-\left\langle u_{0}, \xi\left(\tau_{1}, \cdot\right)\right\rangle_{H^{-1}, H_{0}^{1}}+\langle u(s, \cdot), \xi(s, \cdot)\rangle_{H^{-1}, H_{0}^{1}}+\int_{\tau_{1}}^{s} a(1) \beta(t) \xi_{x}(t, 1) \mathrm{d} t \\
-\int_{s}^{\tau} a(0) \alpha(t) \xi_{x}(t, 0) \mathrm{d} t-\int_{\tau_{1}}^{s} \int_{0}^{1} f(t, x) \xi(t, x) \mathrm{d} x \mathrm{~d} t=0 .
\end{gathered}
$$

See, e.g., [5, Definition 2.36 and Sect. 2.7.1] for this notion of solution, the wellposedness and (4.2). Then using a standard smoothing procedure and the classical maximum principle (see, e.g. [14, Chap. 2]) one gets the following proposition:

Proposition 2. (Maximum principle) Let $\alpha, \beta \in L^{2}\left(\tau_{1}, \tau_{2}\right)$, let $f \in L^{2}\left(\left(\tau_{1}, \tau_{2}\right) \times\right.$ $(0,1))$, and let $u_{0} \in L^{2}(0,1)$ be such that

$$
\alpha \geq 0, \beta \geq 0, f \geq 0, u_{0} \geq 0 .
$$

Then, for every $t \in\left[\tau_{1}, \tau_{2}\right]$,

$$
u(t, \cdot) \geq 0 .
$$


In the next lemma, we derive from this maximum principle an a priori estimate on the solutions of (1.31).

Let us denote by $S(t): L^{2}(0,1) \rightarrow L^{2}(0,1), t \geq 0$, the semigroup generated by the operator $v \mapsto\left(a(x) v_{x}\right)_{x}+c(x) v(t, x)$ with zero Dirichlet boundary condition: for $t \geq 0$ and $u_{0} \in L^{2}(0,1),\left(S(t) u_{0}\right)(x)=v(t, x)$ where $v \in C^{0}\left([0,+\infty) ; L^{2}(0,1)\right)$ is the unique solution of

$$
\begin{cases}v_{t}(t, x)=\left(a(x) v_{x}(t, x)\right)_{x}+c(x) v(t, x) & \text { in }(0,+\infty) \times(0,1), \\ v(t, 0)=v(t, 1)=0 & \text { for } t \in(0,+\infty), \\ v(0, \cdot)=u_{0} . & \end{cases}
$$

Lemma 5. Assume that $F$ satisfies Properties $\left(\mathcal{P}_{1}\right),\left(\mathcal{P}_{2}\right)$ and $\left(\mathcal{P}_{3}\right)$ and that $|F(t, v)| \leq C\|v\|_{L^{2}}^{1 / 2}$ for $(t, v) \in\left[T_{1}, T\right) \times L^{2}(0,1)$ for some $C>0$ and for some $0<T_{1}<T$. Then, there exists $C_{1}>0$ such that, for every $u_{0} \in L^{2}(0,1)$, for every $T_{1} \leq s<s^{\prime}<T$ and for every solution $u \in C^{0}\left(\left[s, s^{\prime}\right] ; L^{2}(0,1)\right)$ of

$$
\begin{cases}u_{t}(t, x)=\left(a(x) u_{x}(t, x)\right)_{x}+c(x) u(t, x) & \text { for }(t, x) \in\left(s, s^{\prime}\right) \times[0,1], \\ u(t, 0)=0, \quad u(t, 1)=F(t, u(t, \cdot)) & \text { for } t \in\left(s, s^{\prime}\right), \\ u(s, \cdot)=u_{0} & \text { for } x \in[0,1],\end{cases}
$$

one has

$$
\left\|u(t, \cdot)-S(t-s) u_{0}\right\|_{L^{2}} \leq C_{1}(t-s)^{1 / 4}\left(1+\left\|u_{0}\right\|_{L^{2}}\right)^{1 / 2} \forall T_{1} \leq s \leq t \leq s^{\prime} .
$$

Proof. Let $v(t, x)$ be the unique solution of the system

$$
\begin{cases}v_{t}(t, x)=\left(a(x) v_{x}(t, x)\right)_{x}+c(x) v(t, x) & \text { in }\left(s, s^{\prime}\right) \times(0,1), \\ v(t, 0)=v(t, 1)=0 & \text { for } t \in\left(s, s^{\prime}\right), \\ v(s, \cdot)=u_{0} . & \end{cases}
$$

Set $w(t, x)=u(t, x)-v(t, x)$. Then

$$
\begin{cases}w_{t}(t, x)=\left(a(x) w_{x}(t, x)\right)_{x}+c(x) w(t, x) & \text { in }\left(s, s^{\prime}\right) \times(0,1), \\ w(t, 0)=0, \quad|w(t, 1)| \leq C\left(\|w(t, \cdot)\|_{L^{2}}^{1 / 2}+\|v(t, \cdot)\|_{L^{2}}^{1 / 2}\right) & \text { for } t \in\left(s, s^{\prime}\right), \\ w(\tau, \cdot)=0 . & \end{cases}
$$

Let $t \in\left(s, s^{\prime}\right]$ be such that $t-s \leq 1$. Set $\tau_{0}=t-s$ and define

$$
W(\tau, x)=\frac{e^{-\frac{\left(1-x+\tau_{0}^{1 / 2}\right)^{2}}{\lambda\left(\tau-s+\tau_{0}\right)}}}{\sqrt{\tau-s+\tau_{0}}} \text { for }(\tau, x) \in[s, t] \times[0,1] .
$$


We have

$$
\begin{aligned}
& \left(a(x) W_{x}(\tau, x)\right)_{x}+c(x) W(\tau, x)-W_{\tau}(\tau, x) \\
& =a(x) W_{x x}(\tau, x)-W_{\tau}(\tau, x)+a_{x}(x) W_{x}(\tau, x)+c(x) W(\tau, x) \\
& \quad=\frac{e^{-\frac{\left(1-x+\tau_{0}^{1 / 2}\right)^{2}}{\lambda\left(\tau-s+\tau_{0}\right)}}}{\sqrt{\tau-s+\tau_{0}}}\left(-\frac{2 a(x)}{\lambda\left(\tau-s+\tau_{0}\right)}+\frac{4 a(x)\left(1-x+\tau_{0}^{1 / 2}\right)^{2}}{\lambda^{2}\left(\tau-s+\tau_{0}\right)^{2}}+\frac{1}{2\left(\tau-s+\tau_{0}\right)}\right. \\
& \left.\quad-\frac{\left(1-x+\tau_{0}^{1 / 2}\right)^{2}}{\lambda\left(\tau-s+\tau_{0}\right)^{2}}+\frac{2\left(1-x+\tau_{0}^{1 / 2}\right)}{\lambda\left(\tau-s+\tau_{0}\right)} a_{x}(x)+c(x)\right) .
\end{aligned}
$$

By choosing $\lambda>0$ small enough, the smallness of $\lambda$ depends only on $a$ and $c$, we have

$$
W_{\tau}(\tau, x) \leq\left(a(x) W_{x}(\tau, x)\right)_{x}+c(x) W(\tau, x) \text { in }(s, t) \times[0,1] .
$$

In what follows, we fix such a constant $\lambda$. Set

$$
\mathcal{W}(\tau, x)=\hat{C} A W(\tau, x) \text { for }(\tau, x) \in(s, t) \times(0,1),
$$

where $\hat{C}$ is a positive constant defined later and

$$
A=(t-s)^{1 / 2}+(t-s)^{1 / 2}\left\|u_{0}\right\|_{L^{2}}^{1 / 2} .
$$

We have, for $\tau \in(s, t)$,

$$
\mathcal{W}(\tau, 1)=\hat{C} A\left(\tau-s+\tau_{0}\right)^{-1 / 2} e^{-\frac{\tau_{0}}{\lambda\left(\tau-s+\tau_{0}\right)}} \geq \hat{C} A[2(t-s)]^{-1 / 2} e^{-1 / \lambda},
$$

which yields, by (4.14),

$$
\mathcal{W}(\tau, 1) \geq C_{\lambda} \hat{C}\left(1+\left\|u_{0}\right\|_{L^{2}}^{1 / 2}\right) .
$$

Here and in what follows $C_{\lambda}$ denotes a positive constant depending only on $\lambda$. Using the fact that, for $m>0$,

$$
\int_{m}^{\infty} e^{-y^{2}} \mathrm{~d} y \leq \frac{\sqrt{\pi}}{2} e^{-m^{2} / 2},
$$

and making a change of variables $y=\left(1-x+\tau_{0}^{1 / 2}\right) / \sqrt{\lambda\left(\tau-s+\tau_{0}\right) / 2}$, we obtain from (4.11) that

$$
\begin{aligned}
\int_{0}^{1}|W(\tau, x)|^{2} \mathrm{~d} x & =\int_{0}^{1} \frac{e^{-\frac{2\left(1-x+\tau_{0}^{1 / 2}\right)^{2}}{\lambda\left(\tau-s+\tau_{0}\right)}}}{\tau-s+\tau_{0}} \mathrm{~d} x \leq \frac{\sqrt{\lambda / 2}}{\sqrt{\tau-s+\tau_{0}}} \int_{1 / \sqrt{\lambda}}^{\infty} e^{-y^{2}} \mathrm{~d} y \\
& \leq \sqrt{\lambda /\left(2 \tau_{0}\right)} e^{-1 /(2 \lambda)}
\end{aligned}
$$

It follows that

$$
\|\mathcal{W}(\tau, \cdot)\|_{L^{2}} \leq \hat{C} A \lambda^{1 / 4}[2(t-s)]^{-1 / 4} e^{-\frac{1}{4 \lambda}},
$$


which implies, by (4.14),

$$
\|\mathcal{W}(\tau, \cdot)\|_{L^{2}} \leq C_{\lambda} \hat{C}(t-s)^{1 / 4}\left(1+\left\|u_{0}\right\|_{L^{2}}^{1 / 2}\right) .
$$

Taking $\hat{C}$ large enough, the largeness of $\hat{C}$ being independent of $s, t$, and $u_{0}$, we derive from (4.16) and (4.19) that

$$
\begin{aligned}
\mathcal{W}(\tau, 1) & \geq 2 C\left(\|\mathcal{W}(\tau, \cdot)\|_{L^{2}}^{1 / 2}+\left\|u_{0}\right\|_{L^{2}}^{1 / 2}\right) \\
& \geq 2 C\left(\|\mathcal{W}(\tau, \cdot)\|_{L^{2}}^{1 / 2}+\|v(\tau, \cdot)\|_{L^{2}}^{1 / 2}\right) \text { for } \tau \in(s, t) .
\end{aligned}
$$

Let us check that, by the maximum principle (Proposition 2), this implies that

$$
|w(t, x)| \leq \mathcal{W}(t, x) \text { for almost every } x \in(0,1) \text {. }
$$

Indeed, the maximum principle leads to (4.21) if

$$
|w(\tau, 1)| \leq \mathcal{W}(\tau, 1) \quad \forall \tau \in[s, t] .
$$

Assume that (4.22) does not hold. Then, there exists $t^{\prime} \in(s, t)$ such that

$$
\begin{aligned}
& |w(\tau, 1)| \leq \mathcal{W}(\tau, 1) \quad \forall \tau \in\left[s, t^{\prime}\right], \\
& \left|w\left(t^{\prime}, 1\right)\right|=\mathcal{W}\left(t^{\prime}, 1\right) .
\end{aligned}
$$

From (4.23) and the maximum principle, we get that

$$
\left|w\left(t^{\prime}, x\right)\right| \leq \mathcal{W}\left(t^{\prime}, x\right) \text { for almost every } x \in(0,1),
$$

which, together with the second line of (4.10) leads to

$$
\left|w\left(t^{\prime}, 1\right)\right| \leq C\left(\left\|\mathcal{W}\left(t^{\prime}, \cdot\right)\right\|_{L^{2}}^{1 / 2}+\left\|v\left(t^{\prime}, \cdot\right)\right\|_{L^{2}}^{1 / 2}\right) .
$$

From (4.20), (4.24) and (4.26), we get a contradiction, which shows that (4.22) and therefore also (4.21) hold. Finally (4.17) and (4.21) yield (4.9) for $t-s \leq 1$. The conclusion in the general case follows immediately from this.

Concerning the existence and uniqueness of the flow $\Phi$, let us prove the following lemma:

Lemma 6. Assume that $F$ satisfies Properties $\left(\mathcal{P}_{1}\right),\left(\mathcal{P}_{2}\right)$, and $\left(\mathcal{P}_{3}\right)$. Let $0 \leq s<T$. There exists $T_{0}=T_{0}(s)>0$ such that, for every $u_{0} \in L^{2}(0,1)$, there exists a unique solution $u \in C^{0}\left(\left[s, s+T_{0}\right) ; L^{2}(0,1)\right)$ of $(1.31)$. Moreover,

$$
\|u(t, \cdot)\|_{L^{2}} \leq C\left\|u_{0}\right\|_{L^{2}} \text { for } t \in\left(s, s+T_{0}\right),
$$

for some positive constant $C=C(s)$ independent of $u_{0}$; moreover, the functions $T_{0}:[0, T) \rightarrow(0,+\infty)$ and $C:[0, T) \rightarrow[0,+\infty)$ can be chosen such that, for every $\delta \in(0, T]$,

$$
\inf \left\{T_{0}(s) ; s \in[0, T-\delta]\right\}>0 \text { and } \sup \{C(s) ; s \in[0, T-\delta]\}<+\infty \text {. }
$$


Proof. Let us first deal with the uniqueness. Let $u_{1}$ and $u_{2}$ be two solutions having the same initial data at time $s$ and defined at least on $\left[s, s^{\prime}\right] \times(0,1)$, with $s<s^{\prime}$. Let $w:\left(s, s^{\prime}\right) \times(0,1) \rightarrow \mathbb{R}$ be defined by $w=u_{2}-u_{1}$. One has, for some $C>0$,

$$
\begin{cases}w_{t}(t, x)=\left(a(x) w_{x}(t, x)\right)_{x}+c(x) w(t, x) & \text { in }\left(s, s^{\prime}\right) \times(0,1) \\ w(t, 0)=0, \quad|w(t, 1)| \leq C\|w(t, \cdot)\|_{L^{2}} & \text { for } t \in\left(s, s^{\prime}\right) \\ w(s, \cdot)=0 & \end{cases}
$$

It suffices to prove that

$$
w(t, \cdot)=0 \text { for } t>s \text { close enough to } s .
$$

We proceed as in the proof of Lemma 5. Fix a positive constant $\lambda$ small such that (4.12) holds. We define $\mathcal{W}$ as in (4.11)-(4.13), with (4.14) now replaced by

$$
A=1 \text {. }
$$

Set $\rho=\left(2(2 C)^{4} \lambda e^{3 / \lambda}\right)^{-1}$. Since

$$
\begin{aligned}
{[2(t-s)]^{-1 / 2} e^{-1 / \lambda} } & \geq 2 C \lambda^{1 / 4}[2(t-s)]^{-1 / 2} e^{-1 /(4 \lambda)} \text { if }(t-s) \\
& \geq\left(2(2 C)^{4} \lambda e^{3 / \lambda}\right)^{-1},
\end{aligned}
$$

it follows from (4.15) and (4.18) that, for $t-s \leq \rho$ and for every $\hat{C}>0$,

$$
\mathcal{W}(\tau, 1) \geq 2 C\|\mathcal{W}(\tau, \cdot)\|_{L^{2}} \text { for } \tau \in(s, t)
$$

(Compare with (4.20).) Then, proceeding as in the proof of (4.21), we get, using the maximum principle,

$$
|w(t, x)| \leq \mathcal{W}(t, x) \text { for almost every } x \in(0,1)
$$

Since $\hat{C}>0$ is arbitrary, it follows from (4.33) that $w(t, \cdot)=0$ for $t-s \leq \rho$. This concludes the proof of the uniqueness.

Let us now establish the existence of $u$ using a fixed point argument. For notational ease, we assume that $s=0$. Set $U_{0}=0$ and, for $n \geq 1$, let $U_{n}$ be the unique solution of

$$
\begin{cases}U_{n, t}(t, x)=\left(a(x) U_{n, x}(t, x)\right)_{x}+c(x) U_{n}(t, x) & \text { in }(0, T) \times(0,1), \\ U_{n}(t, 0)=0, \quad U_{n}(t, 1)=F\left(t, U_{n-1}(t, \cdot)\right) & \text { for } t \in(0, T), \\ U_{n}(t=0, \cdot)=u_{0} & \text { in } L^{2}(0,1) .\end{cases}
$$

Define

$$
W_{n}(t, x)=U_{n+1}(t, x)-U_{n}(t, x) \text { in }(0, T) \times(0,1) .
$$


Let $0<T_{0}<T$. Then, for some positive constant $C$ independent of $n$ and $u_{0}$,

$$
\begin{cases}W_{n, t}(t, x)=\left(a(x) W_{n, x}(t, x)\right)_{x}+c(x) W_{n}(t, x) & \text { in }(0, T) \times[0,1], \\ W_{n}(t, 0)=0 & \text { for } t \in(0, T), \\ \left|W_{n}(t, 1)\right| \leq C\left\|W_{n-1}(t, \cdot)\right\|_{L^{2}} & \text { for } t \in\left(0, T_{0}\right), \\ W_{n}(t=0, \cdot)=0 & \text { in } L^{2}(0,1) .\end{cases}
$$

By the maximum principle (Proposition 2) applied to the function

$$
(t, x) \mapsto R e^{R t} \sup _{\tau \in\left(0, T_{0}\right)}\left\|W_{n-1}(\tau, \cdot)\right\|_{L^{2}} \pm W_{n}(t, x)
$$

with $R>0$ large enough, we have

$$
\left|W_{n}(t, x)\right| \leq M \sup _{\tau \in\left(0, T_{0}\right)}\left\|W_{n-1}(\tau, \cdot)\right\|_{L^{2}} \text { for }(t, x) \in\left(0, T_{0}\right) \times(0,1),
$$

for some $M>0$ independent of $n$ and $u_{0}$. For $0<r<1$, let $\varphi \in C^{1}(\mathbb{R})$ be such that $\varphi(x)=1$ for $x \leq 1-r, \varphi(x)=0$ for $x \geq 1-(r / 2)$ and $\left|\varphi^{\prime}(x)\right| \leq 4 / r$ for $x \in[0,1]$. Multiplying the equation of $W_{n}$ by $W_{n}(t, x) \varphi^{2}(x)$ and integrating by parts, we obtain

$$
\begin{aligned}
& \int_{0}^{1}\left|W_{n}(t, x) \varphi(x)\right|^{2} \mathrm{~d} x+\int_{0}^{t} \int_{0}^{1}\left|\left(W_{n}(t, x) \varphi(x)\right)_{x}\right|^{2} \\
& \quad \leq C \int_{0}^{t} \int_{0}^{1}\left|W_{n}(t, x)\right|^{2}\left|\varphi_{x}(x)\right|^{2} \mathrm{~d} x \mathrm{~d} t .
\end{aligned}
$$

It then follows from (4.36) that, for $0<r<1$,

$$
\begin{aligned}
& \int_{0}^{1-r}\left|W_{n}(t, x)\right|^{2} \mathrm{~d} x+\int_{0}^{t} \int_{0}^{1-r}\left|W_{n, x}(t, x)\right|^{2} \\
& \quad \leq C r^{-2} T_{0} M^{2} \sup _{\tau \in\left(0, T_{0}\right)}\left\|W_{n-1}(\tau, \cdot)\right\|_{L^{2}}^{2} \text { for } t \in\left(0, T_{0}\right) .
\end{aligned}
$$

A combination of (4.36) and (4.37) yields the existence of $\bar{C}$ independent of $r \in$ $(0,1)$, of $n$, and of $u_{0}$, such that

$$
\sup _{\tau \in\left(0, T_{0}\right)}\left\|W_{n}(\tau, \cdot)\right\|_{L^{2}} \leq \bar{C} M\left(r^{-1} T_{0}^{1 / 2}+r^{1 / 2}\right) \sup _{\tau \in\left(0, T_{0}\right)}\left\|W_{n-1}(\tau, \cdot)\right\|_{L^{2}} .
$$

Let us fix $r \in(0,1)$ small enough so that $\bar{C} M r^{1 / 2} \leq 1 / 4$. Then, by choosing $T_{0}$ small enough, we obtain

$$
\sup _{\tau \in\left(0, T_{0}\right)}\left\|W_{n}(\tau, \cdot)\right\|_{L^{2}} \leq \frac{1}{2} \sup _{\tau \in\left(0, T_{0}\right)}\left\|W_{n-1}(\tau, \cdot)\right\|_{L^{2}} .
$$

Hence $\left(U_{n}\right)_{n \in \mathbb{N}}$ is a Cauchy sequence in $C^{0}\left(\left[0, T_{0}\right] ; L^{2}(0,1)\right)$. Let $u \in C^{0}\left(\left[0, T_{0}\right]\right.$; $\left.L^{2}(0,1)\right)$ be its limit. One can easily verify that $u$ is a solution of $(1.31)$ on $\left(0, T_{0}\right)$. Moreover, by (4.38),

$$
\int_{0}^{1}|u(t, x)|^{2} \mathrm{~d} x \leq C\left\|u_{0}\right\|_{L^{2}}^{2} \text { for } t \in\left(0, T_{0}\right) .
$$

Finally, it follows from our proof that (4.28) can also be imposed. 
As a consequence of Lemma 6 we have the following corollary.

Corollary 3. Assume that $F$ satisfies Properties $\left(\mathcal{P}_{1}\right),\left(\mathcal{P}_{2}\right)$ and $\left(\mathcal{P}_{3}\right)$. Let $0 \leq$ $s^{\prime}<T$. Then there exists $C>0$ such that, for every $s \in\left[0, s^{\prime}\right)$ and for every $u_{0} \in L^{2}(0,1)$, there exists a unique solution $u \in C^{0}\left(\left[s, s^{\prime}\right] ; L^{2}(0,1)\right)$ of $(1.31)$ and this solution satisfies

$$
\|u(t, \cdot)\|_{L^{2}} \leq C\left\|u_{0}\right\|_{L^{2}} \text { for every } t \in\left[s, s^{\prime}\right] .
$$

We now assume that $F$ satisfies Properties $\left(\mathcal{P}_{1}\right),\left(\mathcal{P}_{2}\right)$ and $\left(\mathcal{P}_{3}\right)$ and that $(1.33)$ holds. It follows from Corollary 3 that the flow $\Phi$ is well defined on $\left\{\left(t, s, u_{0}\right) \in\right.$ $\left.[0, T) \times[0, T) \times L^{2}(0,1) ; t \geq s\right\}$. In order to prove that

$$
\Phi \text { is well defined on }\left\{\left(t, s, u_{0}\right) \in \mathbb{R} \times \mathbb{R} \times L^{2}(0,1) ; t \geq s\right\},
$$

it only remains to check that, for every $u_{0} \in L^{2}(0,1)$ and for every $s \in[0, T)$,

$$
\Phi\left(t, s, u_{0}\right) \text { is converging in } L^{2}(0,1) \text { as } t \rightarrow T_{-} .
$$

Let $u_{0} \in L^{2}(0,1)$ and let $s \in[0, T)$. Define, for $t \in[s, T), u(t)=\Phi\left(t, s, u_{0}\right)$. It follows from Lemma 5 and Corollary 3 that $t \in[s, T) \mapsto\|u(t)\|_{L^{2}}$ is bounded. Fix $\varepsilon>0$ (arbitrary). By Lemma 5, there exists $\delta>0$ such that

$$
\|u(t)-S(t-T+\delta) u(T-\delta)\|_{L^{2}} \leq \varepsilon \quad \forall t \in[T-\delta, T) .
$$

Let $\delta^{\prime} \in(0, \delta)$ be such that

$$
\left\|S(t-T+\delta) u(T-\delta)-S\left(t^{\prime}-T+\delta\right) u(T-\delta)\right\|_{L^{2}} \leq \varepsilon \quad \forall t, t^{\prime} \in\left[T-\delta^{\prime}, T\right] .
$$

From (4.43) and (4.44), one gets

$$
\left\|u(t)-u\left(t^{\prime}\right)\right\|_{L^{2}} \leq 3 \varepsilon \quad \forall t, t^{\prime} \in\left[T-\delta^{\prime}, T\right) .
$$

This shows that, for every sequence $\left(t_{n}\right)_{n \in \mathbb{N}}$ of real numbers in $(0, T)$ converging to $T$ as $n \rightarrow+\infty$, the sequence $\left(u\left(t_{n}\right)\right)_{n \in \mathbb{N}}$ is a Cauchy sequence in $L^{2}(0,1)$. This concludes the proof of (4.42) and therefore of (4.41).

\section{Proof of Theorem 2}

In this section we prove Theorem 2. Let us first point out that the uniform stability property (1.36) follows from the other conditions. Indeed, we have, by Lemma 5,

$$
\begin{aligned}
& \left\|\Phi\left(t, s, u_{0}\right)\right\|_{L^{2}} \leq C_{1}\left(\left\|u_{0}\right\|_{L^{2}}+(t-s)^{1 / 4}\left(1+\left\|u_{0}\right\|_{L^{2}}\right)^{1 / 2}\right) \\
& \forall 0 \leq \bar{T} \leq s \leq t<T, \quad \forall u_{0} \in L^{2}(0,1) .
\end{aligned}
$$

and, by Corollary 3 ,

$$
\left\|\Phi\left(t, s, u_{0}\right)\right\|_{L^{2}} \leq C_{1}\left\|u_{0}\right\|_{L^{2}} \quad \forall 0 \leq s \leq t<(T+\bar{T}) / 2, \quad \forall u_{0} \in L^{2}(0,1),
$$


for some positive constant $C_{1}$ independent of $u_{0}$. Given $r \geq 0$, let $B(r)$ denote the closed ball of radius $r$ in $L^{2}(0,1)$. For $\varepsilon>0$ (arbitrary), by (5.1) and (5.2) there exists $\delta>0$ such that

$$
\left\|\Phi\left(t, t^{\prime}, u_{0}\right)\right\|_{L^{2}} \leq \varepsilon \quad \forall 0 \leq t^{\prime} \leq t \leq T, \quad \forall u_{0} \in B(\delta) .
$$

Here we also use the fact that

$$
\Phi\left(t, t^{\prime}, u_{0}\right)=\Phi\left(t, \tau, \Phi\left(\tau, t^{\prime}, u_{0}\right)\right) \quad \forall t^{\prime} \leq \tau \leq t, \quad \forall u_{0} \in L^{2}(0,1)
$$

and take $\bar{T}$ close to $T$. Using (5.4) again, we derive from (5.3) the existence of $\eta>0$ such that

$$
\left\|\Phi\left(t, t^{\prime}, u_{0}\right)\right\|_{L^{2}} \leq \varepsilon \quad \forall 0 \leq t^{\prime} \leq t \leq 3 T, \quad \forall u_{0} \in B(\eta),
$$

which, together with (1.26) and (1.35), gives (1.36) provided that we decrease $\eta>0$ if necessary so that $\eta \leq \Gamma$. This implies the uniform stability (1.36).

We next construct $F$. For $n \in \mathbb{N}$, let $\lambda_{n}$ and $t_{n}$ be defined by

$$
\begin{aligned}
& \lambda_{n}=(n+1)^{8} \text { for every } n \in \mathbb{N}, \\
& t_{0}=0, \quad t_{n}=T\left(1-\frac{1}{2 n^{2}}\right) \text { for every } n \in \mathbb{N} \backslash\{0\} .
\end{aligned}
$$

By Corollary 1 , for some $C_{1}$ large enough,

$$
\left\|k_{n}\right\|_{L^{2}} \leq e^{C_{1} \lambda_{n}^{1 / 2}} \text { for every } n \in \mathbb{N} .
$$

We also know from Proposition 1 that there exists some $C_{2}>0$ such that, for $t_{n} \leq t<t_{n+1}$,

$$
\begin{aligned}
\|u(t, \cdot)\|_{L^{2}} & \leq C_{2} e^{-s_{n-1} / 4+C_{2}(n-1)}\left\|u_{0}\right\|_{L^{2}}, \\
|U(t)| & \leq C_{2} e^{-S_{n-1} / 4+C_{2}(n-1)+C_{2} \sqrt{\lambda_{n}}}\left\|u_{0}\right\|_{L^{2}},
\end{aligned}
$$

where $s_{n}=\sum_{k=0}^{n-1} \lambda_{k}\left(t_{k+1}-t_{k}\right)$ for $n \geq 1$ and $s_{0}=1$. It is clear that

$$
\lambda_{n}\left(t_{n+1}-t_{n}\right) \geq C n^{5},
$$

which implies that

$$
\lim _{n \rightarrow+\infty} \frac{\left(t_{n+1}-t_{n}\right) \lambda_{n}}{\sqrt{\lambda_{n+1}}}=+\infty \quad \text { and } \quad \lim _{n \rightarrow+\infty} \frac{s_{n}}{n+\sqrt{\lambda_{n+1}}}=+\infty .
$$

Fix $\alpha$ and $\beta$ two real numbers such that

$$
4<\alpha<\beta<5 .
$$

Let $\left(\mu_{n}\right)_{n \in \mathbb{N}}$ and $\left(v_{n}\right)_{n \in \mathbb{N}}$ be defined by

$$
\mu_{n}:=e^{-n^{\alpha}} \text { and } v_{n}:=e^{-n^{\beta}} \quad \forall n \in \mathbb{N} .
$$


For $n \in \mathbb{N}$, we choose a function $\varphi_{n} \in C^{1}(\mathbb{R})$ such that $0 \leq \varphi_{n} \leq 1, \varphi_{n}(s)=1$ for $s \leq \mu_{n}$ and $\varphi_{n}(s)=0$ if $s \geq 2 \mu_{n}$. Fix $N$ a large (see below) positive integer. We define $F$ in the following way for $t_{n} \leq t<t_{n+1}$,

$$
\begin{aligned}
& F(t, v):=\int_{0}^{1} k_{n}(1, y) v(y) \mathrm{d} y \quad \forall t \in\left[t_{n}, t_{n+1}\right) \text { with } 0 \leq n \leq N-1, \\
& F(t, v):=\varphi_{n}\left(\|v\|_{L^{2}}\right) \int_{0}^{1} k_{n}(1, y) v(y) \mathrm{d} y \quad \forall t \in\left[t_{n}, t_{n+1}\right) \text { with } n \geq N .
\end{aligned}
$$

We derive from (5.8) that, if $N$ is large enough, which is always assumed from now on,

$$
\left\|k_{n}\right\|_{L^{2}} \leq e^{C_{1} \lambda_{n}^{1 / 2}} \leq\left(1 /\left(2 \mu_{n}\right)\right)^{1 / 2} \quad \forall n \geq N .
$$

It follows that, for $t_{n}<t<t_{n+1}$ with $n \geq N$,

$$
|F(t, v)| \leq 2\|v\|_{L^{2}}^{1 / 2}
$$

which gives (1.33). As in (3.7) in the proof of Proposition 1, we get that

$$
\begin{aligned}
& \Phi\left(T, t, u_{0}\right)=0 \quad \forall u_{0} \in B\left(v_{n}\right), \quad \forall t \in\left[t_{N-1}, t_{N}\right] \\
& \Phi\left(T, t, u_{0}\right)=0 \quad \forall t \in\left[t_{n}, t_{n+1}\right) \text { with } n \leq N-2, \quad \forall u_{0} \in B\left(1 / v_{n}\right) .
\end{aligned}
$$

To reach (5.18), we used (5.11). It follows from (5.18) that

$$
\Phi\left(T, t, u_{0}\right)=0 \quad \forall t \in\left[t_{n}, t_{n+1}\right) \text { with } n \leq N-2, \quad \forall u_{0} \in B(\Gamma)
$$

(Let us recall that we always assume $N$ large enough and how large $N$ is depends now on $\Gamma$.) By taking $t_{n}=t$ and $t_{n+1}=t_{n}$ in (3.4) in the proof of Proposition 1 and using (5.11), we have, for $n \geq N$,

$$
\left\|\Phi\left(t_{n}, t, u_{0}\right)\right\|_{L^{2}} \leq \frac{1}{\mu_{n}} \quad \forall u_{0} \in B(\Gamma), \quad \forall t \in\left[t_{n-1}, t_{n}\right] .
$$

From (1.26), (5.1), and (5.18), we get that

$$
\Phi\left(2 T, t, u_{0}\right)=\Phi\left(2 T, T, \Phi\left(T, t, u_{0}\right)\right)=0 \quad \forall \quad t \in\left[t_{N}, T\right], \quad \forall u_{0} \in B\left(1 / \mu_{n}\right)
$$

which, together with (5.19) and (5.20), concludes the proof of Theorem 2.

Remark 7. Feedback laws are important in practice since they are usually much more robust with respect to perturbations (measurement uncertainties, model errors, etc.) than open-loop controls. It would be interesting to study in details this robustness in the case of our feedback law $F$ defined in (5.14)-(5.15). Let us just mention 
that our feedback law $F$ satisfies the following robustness property with respect to model errors. For every $v \in \mathbb{R}, 0 \in L^{2}(0,1)$ remains locally finite-time stable for

$$
\begin{cases}u_{t}(t, x)=\left(a(x) u_{x}(t, x)\right)_{x}+c(x) u(t, x)+v u(t, x) & \text { for }(t, x) \in(s, \tau) \times[0,1], \\ u(t, 0)=0, \quad u(t, 1)=F(t, u(t, \cdot)) & \text { for } t \in(s, \tau) .\end{cases}
$$

More precisely, if $\Phi$ is now the flow associated to (5.22) instead of (1.31), then (1.36) still holds and there exists $\Gamma>0$ (which depends on $v$ ) such that (1.35) holds. Let us point out that, in the context of asymptotic stability instead of finite-time stability, such robustness property does not hold with stationary linear feedback laws if, for example, $a=1$ and $c=0$. More precisely, for every $\mathcal{K} \in L^{2}(0,1)^{*}$, then $0 \in L^{2}(0,1)$ is exponentially unstable for

$$
\begin{cases}u_{t}(t, x)=u_{x x}(t, x)+v u(t, x) & \text { in }(0,+\infty) \times[0,1] \\ u(t, 0)=0, \quad u(t, 1)=\mathcal{K} u(t, \cdot) & \text { for } t \in(0,+\infty)\end{cases}
$$

provided that $\nu \in \mathbb{R}_{+}$is large enough. Indeed, there exists $\theta \in L^{2}(0,1)$ such that

$$
\mathcal{K} v=\int_{0}^{1} \theta(x) v(x) \mathrm{d} x \text { for } v \in L^{2}(0,1) .
$$

Set, for $\mu \in \mathbb{R}$,

$$
u_{\mu}(t, x)=e^{-\mu^{2} t+v t} \sin (\mu x) .
$$

Note that

$$
\lim _{\mu \rightarrow+\infty} \int_{0}^{1} \theta(x) \sin (\mu x) \mathrm{d} x=0 .
$$

Since $v \in \mathbb{R}_{+}$is large enough, it follows that there exists $\mu \in \mathbb{R} \backslash\{0\}$ such that

$$
\mu^{2} \leq v-2 \text { and } \sin \mu=\int_{0}^{1} \theta(x) \sin (\mu x) \mathrm{d} x .
$$

One can easily check that $u_{\mu}$ is a solution of the system

$$
\left\{\begin{array}{c}
u_{t}-u_{x x}=v u \text { for }(t, x) \in(0,+\infty) \times(0,1), \\
u(t, 0)=0, \quad u(t, 1)=\int_{0}^{1} \theta(x) u(t, x) \mathrm{d} x \text { for } t \in(0,+\infty),
\end{array}\right.
$$

and

$$
\lim _{t \rightarrow+\infty} e^{-t}\|u(t, \cdot)\|_{L^{2}(0,1)}=+\infty,
$$

which shows the exponential instability. 


\section{References}

1. Bardos, C., TARTar, L.: Sur l'unicité rétrograde des équations paraboliques et quelques questions voisines. Arch. Rational Mech. Anal., 50, 10-25 (1973)

2. BeKiaris-Liberis, N., Krstic, M.: Compensating the distributed effect of a wave PDE in the actuation or sensing path of multi-input and MIMO LTI systems. Systems Control Lett. 59, 713-719 (2010)

3. Bekiaris-Liberis, N., Krstic, M.: Compensating the distributed effect of diffusion and counter-convection in multi-input and multi-output LTI systems. IEEE Trans. Automat. Control, 56, 637-643 (2011)

4. Cerpa, E., Coron, J.-M.: Rapid stabilization for a Korteweg-de Vries equation from the left Dirichlet boundary condition. IEEE Trans. Automat. Control, 58, 1688-1695 (2013)

5. Coron, J.-M.: Control and nonlinearity, vol. 136 of Mathematical Surveys and Monographs, American Mathematical Soc., Providence, RI, 2007

6. Coron, J.-M., D’AndréA-Novel, B.: Stabilization of a rotating body beam without damping. IEEE Trans. Automat. Control, 43, 608-618 (1998)

7. CoRON, J.-M., Lü, Q.: Local rapid stabilization for a Korteweg-de Vries equation with a Neumann boundary control on the right. J. Math. Pures Appl., 102, 1080-1120 (2014)

8. Coron, J.-M., Lü, Q.: Fredholm transform and local rapid stabilization for a KuramotoSivashinsky equation. J. Diff. Equations, 259, 3683-3729 (2015)

9. Coron, J.-M., Vazquez, R., Krstic, M., Bastin, G.: Local Exponential $H^{2}$ Stabilization of a $2 \times 2$ Quasilinear Hyperbolic System Using Backstepping. SIAM J. Control Optim., 51, 2005-2035 (2013)

10. Di Meglio, F., Vazquez, R., Krstic, M.: Stabilization of a system of $n+1$ coupled first-order hyperbolic linear PDEs with a single boundary input. IEEE Trans. Automat. Control, 58, 3097-3111 (2013)

11. Elharfi, A.: Explicit construction of a boundary feedback law to stabilize a class of parabolic equations. Differential and Integral Equations, 21, 351-362 (2008)

12. Escauriaza, L., Alessandrini, G.: Null-controllability of one-dimensional parabolic equations. ESAIM Control Optim. Calc. Var., 14, 284-293 (2008)

13. FAtTorini, O. H., Russell, D. L.: Exact controllability theorems for linear parabolic equations in one space dimension. Arch. Rational Mech. Anal., 43, 272-292 (1971)

14. Friedman, A.: Partial differential equations of parabolic type, Prentice-Hall, Inc., Englewood Cliffs, N.J., 1964

15. Fursikov, A. V., Imanuvilov, O. Y.:Controllability of evolution equations, vol. 34, Seoul National University, Seoul, 1996

16. Guo, Y.-J. L., Littman, W.: Null boundary controllability for semilinear heat equations. Appl. Math. Optim., 32, 281-316 (1995)

17. Hu, L., Di Meglio, F.: Finite-time backstepping stabilization of $3 \times 3$ hyperbolic systems, IEEE European Control Conference, under review, 2015

18. Hu, L., Di Meglio, F., Vazquez, R., Krstic, M.: Boundary control design of homodirectional and general heterodirectional linear hyperbolic PDEs, Preprint, 2015

19. JonES, B. F.: A fundamental solution for the heat equation which is supported in a strip. J. Math. Anal. Appl., 60, 314-324 (1977)

20. Krstic, M., Guo, B. Z., Balogh, A., Smyshlyaev, A.: Output-feedback stabilization of an unstable wave equation. Automatica, 44, 63-74 (2008)

21. Krstic, M., Kanellakopoulos, I., KoKotovic, P. V.: Nonlinear and adaptive control design, John Wiley \& Sons, Inc., New York, 1995

22. Krstic, M., Smyshlyaev, A.: Backstepping boundary control for first-order hyperbolic PDEs and application to systems with actuator and sensor delays. Systems Control Lett., 57, 750-758 (2008)

23. Krstic, M., Smyshlyaev, A.: Boundary control of PDEs: A course on backstepping designs, vol. 16, SIAM, Philadelphia, 2008 
24. Lebeau, G., Robbiano, L.: Contrôle exact de l'équation de la chaleur. Comm. Partial Differential Equations, 20, 335-356 (1995)

25. Littman, W.: Boundary control theory for hyperbolic and parabolic partial differential equations with constant coefficients. Ann. Scuola Norm. Sup. Pisa Cl. Sci., 5, 567-580 (1978)

26. LIU, W.-J.: Boundary feedback stabilization of an unstable heat equation. SIAM J. Control Optim., 42, 1033-1043 (2003)

27. Liu, W.-J., Krstic, M.: Backstepping boundary control of Burgers's equation with actuator dynamics. Systems Control Lett., 41, 291-303 (2000)

28. Liu, W.-J., KRSTIC, M.: Stability enhancement by boundary control in the KuramotoSivashinsky equation. Nonlinear Anal. Ser. A: Theory Methods, 43, 485-507 (2001)

29. Martin, P., Rosier, L., Rouchon, P.: Null controllability of the heat equation using flatness. Automatica, 50, 3067-3076 (2014)

30. Miller, L.: The control transmutation method and the cost of fast controls. SIAM J. Control Optim., 45, 762-772 (2006)

31. ReIssig, M.: Hyperbolic equations with non-Lipschitz coefficients. Rend. Sem. Mat. Univ. Politec. Torino, 61, 135-181 (2003)

32. Smyshlyaev, A., Cerpa, E., Krstic, M.: Boundary stabilization of a 1-D wave equation with in-domain antidamping. SIAM J. Control Optim., 48, 4014-4031 (2010)

33. Smyshlyaev, A., Krstic, M.: Closed-form boundary state feedbacks for a class of $1-D$ partial integro-differential equations. IEEE Trans. Automat. Control, 49, 2185$2202(2004)$

34. Smyshlyaev, A., Krstic, M.: On control design for PDEs with space-dependent diffusivity or time-dependent reactivity. Automatica, 41, 1601-1608 (2005)

35. Smyshlyaev, A., Krstic, M.: Boundary control of an anti-stable wave equation with anti-damping on the uncontrolled boundary. Systems Control Lett., 58, 617-623 (2009)

36. VAzQuez, R., Krstic, M.: Control of 1-D parabolic PDEs with Volterra nonlinearities, part I: design. Automatica, 44, 2778-2790 (2008)

JEAN-Michel CORON

Laboratoire Jacques-Louis Lions, UMR 7598, UPMC Univ Paris 06, Sorbonne Universités,

4 place Jussieu,

75252 ,

Paris,

France

e-mail: coron@ann.jussieu.fr

and

HoAi-Minh NGUyen

EPFL SB MATHAA CAMA,

Station 8,

1015

Lausanne,

Switzerland

e-mail: hoai-minh.nguyen@epfl.ch

(Received November 6, 2015 / Accepted April 11, 2017)

Published online May 4, 2017 - (C) Springer-Verlag Berlin Heidelberg (2017) 